\title{
Modelling of the B-type binaries CW Cephei and U Ophiuchi
}

\section{A critical view on dynamical masses, core boundary mixing, and core mass}

\author{
C. Johnston ${ }^{1}$, K. Pavlovski ${ }^{2}$, and A. Tkachenko ${ }^{1}$ \\ 1 Instituut voor Sterrenkunde, KU Leuven, Celestijnenlaan 200D, 3001 Leuven, Belgium \\ e-mail: colecampbell. johnston@kuleuven. be \\ 2 Department of Physics, Faculty of Science, University of Zagreb, Bijenička cesta 32, 10000 Zagreb, Croatia \\ Received 8 February 2019 / Accepted 15 May 2019
}

\begin{abstract}
Context. Intermediate-mass stars are often overlooked. They are not supernova progenitors, but still host convective cores and complex atmospheres that require computationally expensive treatment. This means that there is a general lack of this class of stars modelled by state-of-the-art stellar structure and evolution codes.

Aims. We used high-quality spectroscopy to update the dynamically obtained stellar parameters and to produce a new evolutionary assessment of the bright B0.5+B0.5 and B5V+B5V binary systems CW Cep and U Oph.

Methods. We used new spectroscopy obtained with the Hermes spectrograph to revisit the photometric binary solution of the two systems. The updated mass ratio and effective temperatures are incorporated to obtain new dynamical masses for the primary and secondary. With these data we performed evolutionary modelling using isochrone-clouds to investigate the core properties of these stars.

Results. We report the first abundances for CW Cep and U Oph, and we report an updated dynamical solution for the two systems. We find that we cannot uniquely constrain the amount of core boundary mixing in any of the stars we consider. Instead, we report their core masses and compare our results to previous studies.

Conclusions. We find that the per-cent level precision on fundamental stellar quantities are accompanied with core mass estimates to a precision between $\sim 5 \%$ and $15 \%$. We find that differences in analysis techniques can lead to substantially different evolutionary modelling results, which calls for the compilation of a homogeneously analysed sample to draw inferences on internal physical processes.
\end{abstract}

Key words. binaries: eclipsing - stars: fundamental parameters - stars: interiors - stars: evolution - stars: individual: CW Cep stars: individual: U Oph

\section{Introduction}

The model-independent estimates of the absolute dimensions of and distances to stars provided by eclipsing binary systems serve as a fundamental calibrator in modern astrophysics. In the best cases, these systems offer dynamical mass and radius estimates to better than one per cent (Torres et al. 2010). Such precise measurements combined with the powerful constrains of co-evolution and identical initial chemical composition have allowed the thorough investigation of the importance of rotation in stellar evolutionary theory (Brott et al. 2011a,b; Ekström et al. 2012, 2018; de Mink et al. 2013; Schneider et al. 2014), the calibration of pre- through post-main-sequence evolution (Torres et al. 2013; Higl \& Weiss 2017; Beck et al. 2018a; Kirkby-Kent et al. 2018), the critical investigation of magnetic fields in stars (Takata et al. 2012; Grunhut et al. 2013; Torres et al. 2014b; Pablo et al. 2015; Kochukhov et al. 2018; Wade et al. 2019), the calibration of distances (Guinan et al. 1998; Ribas et al. 2000a, 2005; Hensberge et al. 2000; Bonanos et al. 2006; Pietrzyński et al. 2013; Gallenne et al. 2016; Suchomska et al. 2019), the investigation of abundances and rotational velocities (Pavlovski \& Hensberge 2005; Pavlovski \& Southworth 2009; Pavlovski et al. 2009, 2018; Simón-Díaz et al. 2017), and the calibration of asteroseismic modelling (De Cat et al. 2000, 2004;
Aerts \& Harmanec 2004; Schmid et al. 2015; Schmid \& Aerts 2016; Beck et al. 2018a,b; Johnston et al. 2019). Additionally, the development of such precise measurements has led to the reported systematic discrepancy between masses obtained via dynamics or empirical spectral relations and fitting theoretically calculated evolutionary tracks (Herrero et al. 1992; Ribas et al. 2000b; Tkachenko et al. 2014). This discrepancy has served as the centrepiece of intense debate over the importance of convective core boundary mixing in stellar evolution theory (Ribas et al. 2000b; Torres et al. 2010, 2014a; Tkachenko et al. 2014; Stancliffe et al. 2015; Claret \& Torres 2018; Constantino \& Baraffe 2018; Johnston et al. 2019).

In general, both element and angular momentum transport processes throughout a star are poorly calibrated (Aerts et al. 2019). It is a well-known shortcoming of most $1 \mathrm{D}$ theoretical descriptions of convection that convective boundaries are not well described (Hirschi et al. 2014). Proposed as a means to remedy this shortcoming, convective core overshooting was included as a way to increase near-core mixing in evolutionary models; it is now highly debated, with several competing studies claiming that models with and without overshooting can reproduce observed binaries across different mass ranges and evolutionary stages (Andersen et al. 1990; Schroder et al. 1997; Pols et al. 1997; Claret 2007; Stancliffe et al. 2015; Claret \& Torres 2016, 2017; 
Higl \& Weiss 2017; Constantino \& Baraffe 2018). Convective core overshooting is a phenomenon theoretically predicted in intermediate- to high-mass stars with a convective core, where the inertia of a convectively accelerated mass element propels said mass element beyond the convective boundary described by the Schwarzchild stability criterion into the stably stratified radiative region (Zahn 1977; Roxburgh 1978; Zahn 1991; Maeder 2009). The mathematical form of the implementation into stellar evolutionary codes is not universally agreed upon, with different forms having been successfully used to describe both binary (Ribas et al. 2000b; Guinan et al. 2000; Tkachenko et al. 2014; Claret \& Torres 2016, 2017) and asteroseismic observations (Briquet et al. 2007; Moravveji et al. 2015, 2016; Van Reeth et al. 2016; Johnston et al. 2019). To date, two such descriptions have been implemented in 1D stellar evolution codes: (i) convective penetration where the temperature gradient in the overshoot region is adiabatic, $\nabla_{\mathrm{T}}=\nabla_{\mathrm{ad}}$, and (ii) diffusive overshooting where the temperature gradient is radiative, $\nabla_{\mathrm{T}}=\nabla_{\text {rad }}$. This difference results in a fully chemically and thermally mixed extended region in the case of penetration, effectively meaning the core is extended and thus more massive. In the case of diffusive overshooting, the extended region is only partially chemically mixed, and any increase in core mass is due to the transport of chemicals into the convective core via this chemical mixing. In either case, the convective core will thus have more hydrogen available to burn (or He in the He-core burning phase), thus extending the main sequence (MS) lifetime of the star and having a pronounced effect on the morphology of evolutionary tracks. Alternatively, some studies have used near-core rotational mixing to enhance the core mass, effectively producing the same situation where more rotational mixing corresponds to a more massive core. We note that in 1D diffusive codes, the implementations of overshooting and rotational mixing seem different, but both are able to function as a proxy for the total amount of near-core mixing, whatever the physical cause, and that both prescriptions contain uncalibrated parameters. We adopt the approach of using overshooting as a general proxy for the total amount of near-core mixing, whatever its physical cause (rotation, convection, magnetism, waves). The mass discrepancy reported between either spectroscopic (Herrero et al. 1992) or dynamical masses (Guinan et al. 2000; Ribas et al. 2000b; Claret 2007; Tkachenko et al. 2014) and evolutionary masses has traditionally been resolved by increasing the amount of overshooting in a stellar model. This increase in overshooting effectively increases the core mass at a given age, mimicking a more massive star.

It was theoretically outlined that the extent of an overshooting region would be limited by the total energy (mass) of the core (Roxburgh 1992), and hence the mass of the star. This theoretical prediction has been investigated by numerous studies, some claiming no significant mass dependence (Schroder et al. 1997; Pols et al. 1997; Stancliffe et al. 2015; Constantino \& Baraffe 2018), while others claim a statistically significant mass dependence (Claret 2007; Claret \& Torres 2016, $2017,2018,2019)$. Yet another body of work suggests caution at the ability to constrain overshooting from classical observable quantities given the sensitivity of the data and methodologies (Valle et al. 2016; Higl \& Weiss 2017; Valle et al. 2017, 2018; Johnston et al. 2019; Constantino \& Baraffe 2018). On the theoretical side, Valle et al. (2016) studied the ability of models to uniquely describe a set of observables, revealing an inability to uniquely constrain overshooting. This result was supported by the findings of Valle et al. (2018) and Constantino \& Baraffe (2018) who show that traditional observed quantities do not provide enough discriminating power to uniquely constrain overshooting, with Constantino \& Baraffe (2018) being unable to reproduce the mass dependence of overshooting reported by Claret \& Torres (2016). Furthermore, Johnston et al. (2019) showed that even with the inclusion of asteroseismic information, the extent of overshooting, stellar mass, and age cannot be uniquely constrained when properly accounting for correlated nature of stellar model parameters. Instead, Johnston et al. (2019) suggest that the mass and radius of the convective core should be reported and considered in place of the overshooting.

In this paper, we follow the paradigm of Johnston et al. (2019) to investigate the ability of well-detached double-lined eclipsing binaries (EBs) to probe the core mass. Additionally, we investigate the sparsely sampled mass range of 4-6 $M_{\odot}$, not covered by previous studies (Claret \& Torres 2016, 2017; Pols et al. 1997; Higl \& Weiss 2017). We revisit the intermediate- to highmass double-lined EBs CW Cep and U Oph with new spectroscopy and radial velocities to obtain updated mass and radii estimates. In Sect. 2, we provide an overview of the two systems, including past modelling efforts. In Sect. 3 we discuss the new spectroscopy and the newly determined orbital elements from spectral disentangling, and in Sect. 4 we discuss the determination of spectroscopic quantities from the disentangled spectra. In Sect. 5 we detail the modelling procedure and results for the two systems with the mass ratio fixed as derived in the previous section. Section 6 covers our evolutionary modelling procedure. In Sects. 6.2 and 7 we discuss the newly determined mass and radii estimates for each system and the modelling results, and we place them in the context of the larger modelling efforts of the community. Following the results of Constantino \& Baraffe (2018) and Johnston et al. (2019), we report and discuss the estimated core mass and overshooting from our modelling procedure.

\section{Literature overview of CW Cep and U Oph}

\subsection{CW Cephei}

The detached double-lined EB CW Cephei (HD 218066, $V=7.6 \mathrm{mag}$ ) is an intensively studied system. The component masses have reported values in the ranges $M_{1}=$ 11.82-13.49 $M_{\odot}$ and $M_{2}=11.09-12.05 M_{\odot}$ (Popper 1974, 1980; Clausen \& Gimenez 1991; Han et al. 2002), placing this system at the lower end of the high-mass sequence. This spread in masses results in an uncertainty of $\sim 13 \%$ compared to the median value (solution $b$ by Han et al. 2002). The quality of the photometric light curve solution, in particular the determination of the masses and radii, has been restricted by uncertainty on the mass and light ratio, respectively. This problem has been extensively discussed by Clausen \& Gimenez (1991), who found that the spread in radii ratios is also accompanied by a significant spread in the sum of the radii. Subsequent analysis of their own new photometry by Han et al. (2002) and Erdem et al. (2004) did not settle issue as they used a different methodology from Clausen \& Gimenez (1991). Specifically, Han et al. (2002) did not prefer the photographically determined light ratio over that returned from the light curve modelling, and Erdem et al. (2004) allowed for the possibility of asynchronous rotation in the components, which alters the light ratios derived from photometric modelling. Comparing the radii derived by different previous analyses (a complete set of the references are given in Han et al. 2002), a spread of $\sim 8 \%$ is found.

Apsidal motion was detected in CW Cep by Nha (1975) with improvements to the apsidal period made by Han et al. (2002), 
Erdem et al. (2004), and Wolf et al. (2006). The last authors settled the apsidal period to $U=46.2 \pm 0.4 \mathrm{yr}$, with an eccentricity of $e=0.0246$. The relatively short apsidal period, coupled with the brightness of the system have made it an ideal target for dynamical and evolutionary studies. Currently, the nature of the mechanism that drives the apsidal motion is not well understood. New space-based, high-precision, high duty cycle observations from the NASA TESS mission (Ricker et al. 2015) will provide hitherto unseen constraints on the apsidal motion observed in this system.

Due to a distinct lack of constraints on the metallicity of CWCep, the unique determination of evolutionary models for CW Cep has proven difficult (Clausen \& Gimenez 1991). To date, several age estimates for CW Cep exist, with Clausen \& Gimenez (1991) reporting an age of $\tau=(10 \pm 1) \mathrm{Myr}$, placing both components in the first half of the main sequence. In their fitting work, Ribas et al. (2000c) derived a much younger system with $\tau=4.6 \pm 0.5 \mathrm{Myr}$. However, Ribas et al. (2000c) varied the metallicity and helium content, which introduces a near-perfect degeneracy with age and mass in evolutionary modelling. Thus, their solution with $\tau=4.6 \pm 0.5 \mathrm{Myr}, Z=$ $0.023 \pm 0.007$, and a high helium content, albeit with a large uncertainty $Y=0.298 \pm 0.101$, is entirely consistent with that of Clausen \& Gimenez (1991) given this degeneracy. Recently, CW Cep has been modelled by Schneider et al. (2014), who used a Bayesian modelling framework wrapped around Bonn evolutionary tracks to derive an age of $\sim 6 \mathrm{Myr}$, with a best fit for an initial rotational velocity of $v \sin i=520 \mathrm{~km} \mathrm{~s}^{-1}$ for both components. It should be noted that Schneider et al. (2014) used a less massive solution in their modelling than Ribas et al. (2000c), by $\sim 0.5 M_{\odot}$ for the primary and $\sim 0.3 M_{\odot}$ for the secondary, and fixed the metallicity of their tracks to solar. Furthermore, Blaauw et al. (1959) identified CW Cep to be a member of the Cep OB3, one of the smaller associations in the Orion arm. Blaauw (1961) also indicated that this association is composed of two subgroups. CW Cep is located in the older subgroup for which Clausen \& Gimenez (1991) found an average age of about $10 \mathrm{Myr}$, in perfect agreement with the age they obtained for CW Cep. However, in a comprehensive study of new homogeneous $U B V R I$ photometry and membership, Jordi et al. (1996) obtained ages of 5.5 and 7.5 Myr for the two subgroups in disagreement with the ages derived by both Clausen \& Gimenez (1991) and Schneider et al. (2014).

CW Cep is also characterised as an intrinsically variable polarised object (Elias et al. 2008). Both CW Cep and another early B+B binary system AH Cep, were observed with the Chandra X-ray Telescope in search of evidence of a wind-wind collision (Ignace et al. 2017). Although CW Cep and AH Cep are comprised of stars with similar properties (cf. Pavlovski et al. 2018), X-rays were only detected for AHCep, even though it is nearly a factor of 2 further away than CW Cep. The authors could not disentangle whether the X-rays detected from AH Cep were caused by colliding winds, or perhaps from magnetic activity originating in one of the other components of the quadruple system of AH Cep (Ignace et al. 2017).

\subsection{U Ophiuchi}

U Oph (HD 156247, $V=5.92 \mathrm{mag}$ ) is a detached double-lined EB comprised of two B5V components. As in the case of CW Cep, the dynamical solution of U Oph suffers from uncertainties on the light and mass ratios from spectral analysis. The reported masses for $\mathrm{U} O p h$ are in the ranges $M_{1}=4.93-5.27 M_{\odot}$ and $M_{2}=4.56-4.78 M_{\odot}$, whereas the reported radii $R_{1}=$
3.29-3.48 $R_{\odot}$ and $R_{2}=3.01-3.11 R_{\odot}$ (Holmgren et al. 1991; Vaz et al. 2007; Wolf et al. 2006; Budding et al. 2009). This represents an uncertainty of $\sim 7 \%$ and $\sim 5 \%$ on $M_{1}$ and $M_{2}$ and an uncertainty of $\sim 6 \%$ and $\sim 3 \%$ on $R_{1}$ and $R_{2}$ when compared to the most recent solution by Budding et al. (2009). Additionally, a wide range of effective temperatures has been reported for both components, with differences up to $3000 \mathrm{~K}$ (Clements \& Neff 1979; Eaton \& Ward 1973; Holmgren et al. 1991; Andersen et al. 1990; Budding et al. 2009).

A majority of the past light curve solutions are based on either the unfiltered photoelectric measurements of Huffer \& Kopal (1951), the OAO-2 spacecraft photometery of Eaton \& Ward (1973), or both. However, the work of Vaz et al. (2007) and Budding et al. (2009) relies on new photometric and spectroscopic data. While all of these analyses use different modelling methodologies, codes, and assumptions, they produce derived quantities within a rather small range, as discussed above, and with high precision, which is promising. $\mathrm{U}$ Oph displays a very rapid apsidal motion with a period of $U \approx 20 \mathrm{yr}$ attributed to a distant third body (Koch \& Koegler 1977; Kaemper 1986; Wolf et al. 2002). The apsidal motion has been studied thoroughly with several proposed apsidal periods, some as large as $55 \mathrm{yr}$ (Frieboes-Conde \& Herczeg 1973; Panchatsaram 1981; Wolf et al. 2002; Vaz et al. 2007). Several recent studies have tried to constrain the nature of the tertiary component, reporting an orbital period of $P_{3} \approx 38 \mathrm{yr}$ and $M_{3} \approx 1 M_{\odot}$ (Kaemper 1986; Wolf et al. 2002; Vaz et al. 2007; Budding et al. 2009).

Largely due to uncertainties in its metallicity, there have been several discrepant ages reported for U Oph. Holmgren et al. (1991) first reported an age of $\sim 40 \mathrm{Myr}$ for U Oph when compared to evolutionary tracks without overshooting, and an age $\sim 63 \mathrm{Myr}$ when compared to evolutionary tracks with overshooting. Later, Vaz et al. (2007) compared their solution to evolutionary tracks of different metallicities, considering the apsidal constant as an additional constraint in their modelling and found the best agreement with isochrones for $\sim 40 \mathrm{Myr}, \sim 52 \mathrm{Myr}$, and $\sim 62 \mathrm{Myr}$ calculated at $Z=0.02,0.017$, and 0.01 , respectively. Budding et al. (2009) perform their own evolutionary analysis, again with different codes and solutions compared to the previous evolutionary modelling attempts, and arrive at an average age estimate of $\sim 38 \mathrm{Myr}$ between the two components for tracks calculated at $Z=0.02$. The authors also note that a younger solution is found at $\sim 30 \mathrm{Myr}$ from tracks calculated at $Z=0.03$. More recently, Schneider et al. (2014) model U Oph with the Bonnsai code, assuming rotational mixing in their models (calculated at $Z=0.014$ ) and find an average age of $\sim 41 \mathrm{Myr}$ for the system. Budding et al. (2009) provide a comprehensive and detailed discussion of $U$ Oph, to which we refer the reader for additional information.

\section{Orbital elements from new high-resolution spectroscopy}

For both CW Cep and U Oph, we obtained a new series of highresolution échelle spectra using the High-Efficiency and highResolution Mercator Échelle Spectrograph HERMEs on the $1.2 \mathrm{~m}$ Mercator telescope at the Observatorio del Roque de los Muchachos, La Palma, Canary Islands, Spain. The HERmes spectrograph covers the entire optical and near-IR (NIR) wavelength range (3700-9100 $\AA$ ) with a spectral resolution of $R=85000$ (Raskin et al. 2011). CW Cep was observed a total of 18 times over 13 nights. Three observations were performed in January 
Table 1. Orbital parameters determined by method of spectral disentangling.

\begin{tabular}{lccc}
\hline \hline Param. & Unit & CW Cep & U Oph \\
\hline$P$ & $\mathrm{~d}$ & 2.72913159 & 1.67734590 \\
$T_{\text {per }}$ & $\mathrm{d}$ & $57608.72 \pm 0.05$ & - \\
$e$ & - & $0.0298 \pm 0.0008$ & 0. \\
$\omega$ & $\mathrm{deg}$ & $218.7 \pm 5.7$ & 90. \\
$K_{\mathrm{A}}$ & $\mathrm{km} \mathrm{s}^{-1}$ & $211.1 \pm 0.4$ & $181.1 \pm 0.6$ \\
$K_{\mathrm{B}}$ & $\mathrm{km} \mathrm{s}^{-1}$ & $230.2 \pm 0.4$ & $200.6 \pm 0.8$ \\
\hline$q$ & - & $0.917 \pm 0.002$ & $0.903 \pm 0.005$ \\
$M_{\mathrm{A}} \sin ^{3} i$ & $M_{\odot}$ & $12.66 \pm 0.05$ & $5.08 \pm 0.04$ \\
$M_{\mathrm{B}} \sin ^{3} i$ & $M_{\odot}$ & $11.61 \pm 0.04$ & $4.59 \pm 0.04$ \\
$a \sin i$ & $R_{\odot}$ & $23.78 \pm 0.03$ & $12.65 \pm 0.03$ \\
\hline
\end{tabular}

Notes. The periods were fixed from photometry in these calculations.

2015 with the remaining 15 in August 2016. The argument of periastron progressed $\sim 10^{\circ}$ between these two subsets, and less than one degree within either subset. U Oph, was observed 11 times over 10 nights from April to August 2016, during which time the argument of periastron progressed $\sim 4^{\circ}$. The resulting spectra have an average $\mathrm{S} / \mathrm{N}$ of 110 in a range 51-144 and 145 in a range 117-163 for CW Cep and U Oph, respectively.

The basic reduction of the spectra was performed with the HERMES pipeline software package. This pipeline delivers merged, un-normalised spectra. Therefore, before disentangling the spectra, we performed normalisation via spline function.

Spectral disentangling (hereafter SPD) models the Doppler shift of spectral lines from a time-series of double-lined stellar spectra to determine the spectroscopic orbital elements and to simultaneously reconstruct the individual spectra of the components (Simon \& Sturm 1994). Since the orbital elements are directly optimised in SPD, the determination of radial velocities for each individual exposure is side-stepped. This removes the dependence on template spectra, which are commonly used in the cross-correlation function $(\mathrm{CCF})$ radial velocity $(\mathrm{RV})$ determination method; these templates are often a source of systematic error due to mismatches between the spectral type of the star and that of the template (Hensberge \& Pavlovski 2007). Moreover, the resulting disentangled spectra of each component have an increased $\mathrm{S} / \mathrm{N}$ compared to single-shot spectra, since disentangling acts as co-addition of the input spectra (cf. Pavlovski \& Hensberge 2010). We employ the FDBinary code (Ilijic et al. 2004), which performs SPD in Fourier space in order to efficiently solve the large and over-determined system of linear equations represented by the data via discrete Fourier transforms to the spectra (Hadrava 1995).

FDBINARY calculates the RV curve for each component using the standard set of orbital elements: period $P_{\text {orb }}$, time of periastron passage $T_{\text {per }}$, eccentricity $e$, the argument of periastron $\omega_{0}$, and the semi-amplitudes of the RVs variations for the components $K_{1}$ and $K_{2}$. FDBINARY simultaneously optimises all orbital parameters across the entire set of spectra utilising the SIMPLEX algorithm. Although the Balmer lines dominate the optical spectra of hot stars, these lines are broad and usually cover a majority of a single échelle order; any imperfections in the order-merging and normalisation procedure would thus propagate into the optimisation and affect both the orbital elements and the resulting disentangled component spectra. Therefore, helium and metal lines are more suitable for our purposes. The resulting optimised orbital parameters for CW Cep and U Oph are listed in Table 1.
The orbital parameters of CW Cep and U Oph have been derived from fitting RVs in numerous previous studies. For CW Cep, Stickland et al. (1992) determined $K_{\mathrm{A}}=210.6 \pm$ $1.3 \mathrm{~km} \mathrm{~s}^{-1}$, and $K_{\mathrm{B}}=229.9 \pm 1.6 \mathrm{~km} \mathrm{~s}^{-1}(q=0.92 \pm 0.1)$ by fitting RVs extracted from three days of IUE spectra using a $\mathrm{CCF}$ method. However, since they had a relatively small number of spectra (21), the authors chose to fix the eccentricity to $e=0.0293$ following Clausen \& Gimenez (1991). Alternatively, Popper \& Hill (1991) obtained $K_{\mathrm{A}}=210 \pm 2 \mathrm{~km} \mathrm{~s}^{-1}$ and $K_{\mathrm{B}}=235 \pm 2 \mathrm{~km} \mathrm{~s}^{-1}(q=0.89 \pm 0.01)$ by fitting RVs obtained via the CCF method from digitised plate spectra of CW Cep obtained with the Lick Observatory $3 \mathrm{~m}$ telescope. The value for $K_{\mathrm{B}}$ derived by Popper \& Hill (1991) is substantially higher than that obtained by Popper (1974) who used the very same data, but employed the oscilloscopic method to determine RVs as opposed to the CCF method that was used by Popper \& Hill (1991). By comparison, our results for CW Cep, listed in Table 1, place our estimates within $1 \sigma$ of the solution presented by Stickland et al. (1992) and within $2 \sigma$ of Popper \& Hill (1991).

Popper \& Hill (1991) also re-fit the orbital parameters of U Oph on RVs determined via CCF from Lick Observatory plate spectra, reporting $K_{\mathrm{A}}=183 \pm 2.5 \mathrm{~km} \mathrm{~s}^{-1}$, and $K_{\mathrm{B}}=$ $195 \pm 3 \mathrm{~km} \mathrm{~s}^{-1}(q=0.94 \pm 0.02)$. Additionally, Holmgren et al. (1991) reported $K_{\mathrm{A}}=182 \pm 1 \mathrm{~km} \mathrm{~s}^{-1}$ and $K_{\mathrm{B}}=197 \pm 1 \mathrm{~km} \mathrm{~s}^{-1}$ $(q=0.924 \pm 0.007)$ from $31 \mathrm{RV}$ measurements extracted via CCF from 31 Reticon spectra obtained at the Dominion Astrophysical Observatory (DAO) $1.2 \mathrm{~m}$ telescope. Later, Vaz et al. (2007) obtained slightly different estimates of $K_{\mathrm{A}}=182.7 \pm 1.2 \mathrm{~km} \mathrm{~s}^{-1}$ and $K_{\mathrm{B}}=203.3 \pm 1.6 \mathrm{~km} \mathrm{~s}^{-1}(q=0.90 \pm 0.01)$ from 34 plate spectra obtained by the ESO $1.5 \mathrm{~m}$ telescope. Until this work the only results based on échelle spectra had been presented by Budding et al. (2009) who found $K_{\mathrm{A}}=180.0 \pm 1.3 \mathrm{~km} \mathrm{~s}^{-1}$ and $K_{\mathrm{B}}=202.7 \pm 1.2 \mathrm{~km} \mathrm{~s}^{-1}(q=0.89 \pm 0.01)$ from $30 \mathrm{RV}$ measurements determined via CCF from spectra obtained with the HERCULES spectrograph attached to the $1 \mathrm{~m}$ Canterbury University McLellan Telescope located at Mt. John University Observatory in New Zealand. Our results are in rough agreement with the literature values, but highlight the increased precision provided by SPD which inherently minimises uncertainties presented by line blending and template mismatches that plague $\mathrm{CCF}$ techniques.

\section{Atmospheric parameters from disentangled spectra}

\subsection{CW Cep}

CW Cep consists of two early-B spectral type stars with $T_{\text {eff }} \sim 28000 \mathrm{~K}$ (Popper 1974, 1980; Clausen \& Gimenez 1991; Han et al. 2002). These temperature estimates place both components in the temperature range where the strength of He II lines starts to grow, thus allowing us to obtain precise effective temperature estimates through fine-tuning the helium ionisation balance. As such, we apply the same methodology as described in Pavlovski et al. (2018), which we briefly summarise here.

An observed spectrum of a binary is a composite of spectra of the two components, which means that the disentangled spectra are equal to the spectra of the intrinsic components multiplied by the respective light factors, i.e. the components' fractional light contribution to the total light of a binary system, such that their co-addition reaches unity in the continuum. Generally, the fractional light contribution of each component can be determined either in the light curve analysis, or extracted from disentangled spectra. In the case of partially eclipsing binary systems where the components have similar radii, the light ratios 
Table 2. Atmospheric parameters derived from an optimal fitting of re-normalised disentangled spectra for the components of CW Cep and U Oph.

\begin{tabular}{lcccc}
\hline \hline Component & $\begin{array}{c}T_{\text {eff }} \\
{[\mathrm{K}]}\end{array}$ & $\begin{array}{c}\log g \\
{[\mathrm{dex}]}\end{array}$ & $\begin{array}{c}\xi_{\mathrm{t}} \\
{\left[\mathrm{km} \mathrm{s}^{-1}\right]}\end{array}$ & $\begin{array}{c}v \sin i \\
{\left[\mathrm{~km} \mathrm{~s}^{-1}\right]}\end{array}$ \\
\hline CW Cep A & $28300 \pm 460$ & 4.079 & $2.0 \pm 0.5$ & $105.2 \pm 2.1$ \\
CW Cep B & $27550 \pm 420$ & 4.102 & $1.5 \pm 0.5$ & $96.2 \pm 1.9$ \\
U Oph A & $16580 \pm 180$ & 4.073 & 2.0 & $110 \pm 6$ \\
U Oph B & $15650 \pm 200$ & 4.131 & 2.0 & $108 \pm 6$ \\
\hline
\end{tabular}

Notes. For CW Cep a grid of NLTE synthetic spectra was used, whilst for U Oph a grid of LTE synthetic spectra were used. The quantities given without the uncertainties were fixed in the calculation.

are degenerate with the radii ratio and inclination. Therefore, it is advantageous to use the light ratio derived from disentangled spectra in the light curve modelling. We follow an iterative approach, where we first vary both the light factors and surface gravities, and then impose the light factors derived from spectroscopy as priors in our light curve modelling. To obtain atmospheric parameters, an optimised fit to the disentangled spectra of each component, which are re-normalised by their lightfactor, is performed over a grid of pre-calculated non-local thermodynamic equilibrium (NLTE) models using the STARFIT code (Tamajo et al. 2011; Kolbas et al. 2014). These theoretical NLTE spectra were calculated using ATLAS9 model atmospheres and the NLTE spectral synthesis suite DETAIL/sURFACE (Giddings 1981; Butler et al. 1984). The synthetic spectra grid used in the optimisation contains models with $T_{\text {eff }} \in 15000-32000 \mathrm{~K}$, and $\log g \in 3.5-4.5 \mathrm{dex}$, and solar metallicity $[\mathrm{M} / \mathrm{H}]=0$. However, we are able to fix the $\log g$ for each component according to the values listed in Table 5, since high-precision, independent estimates for the surface gravities were derived from the light curve modelling. Fixing the surface gravity effectively lifts the degeneracy between the effective temperature and surface gravity, and enables us to use the Balmer lines as constraining information in our fit found by the helium ionisation balance. By fixing the surface gravity and micro-turbulence per component, we reduce the optimisation to eight free parameters: the effective temperature $T_{\text {eff }}$ per component, projected rotational velocity $v \sin i$ per component, a relative Doppler shift between disentangled spectra, and laboratory reference frame, and the light-factors of the disentangled components. The optimisation across this parameter space is performed via a genetic algorithm modelled after that of the PIKAIA subroutine by Charbonneau (1995), with the errors calculated via a Markov chain Monte Carlo technique as described by Ivezić et al. (2014) and implemented by Kolbas et al. (2014). The optimisation was carried out over the spectral segment from $4000-4700 \AA$, and includes the Balmer lines $\mathrm{H} \gamma$ and $\mathrm{H} \delta$, in addition to helium lines from both ionisation stages. Other spectral lines were masked. Due to the strong interstellar absorption band which effects the red wing of the $\mathrm{H} \beta$ line, we were unable to use this spectral segment which covers the $y$ filter. However, since the effective temperatures of CW Cep A and B are similar, the wavelength dependence of the light-ratio is very small. The final analysis with fixed surface gravities and variable light ratios returned $T_{\text {eff }, \mathrm{p}}=28300 \pm 460 \mathrm{~K}$, and $T_{\text {eff }, \mathrm{s}}=27550 \pm 420 \mathrm{~K}$ with light factors of $0.565 \pm 0.005$ and $0.425 \pm 0.005$, for the primary and secondary, respectively. We note that these light factors are the same as those determined from the initial iteration, within the errors. The full optimised parameters are listed in Table 2. The best fits
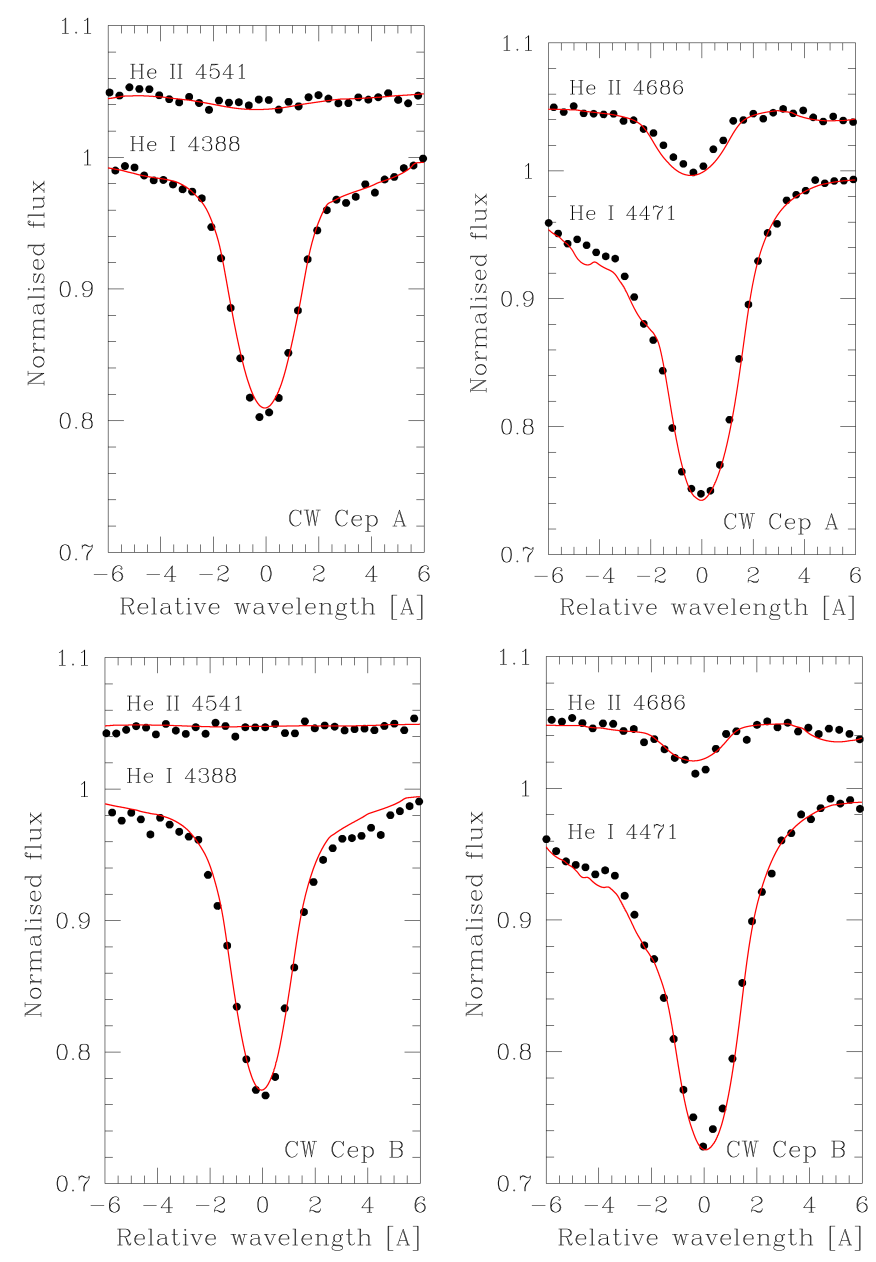

Fig. 1. Determination of the $T_{\text {eff }}$ for the components of CW Cep: the primary, component A (upper panels), the secondary, component $\mathrm{B}$ (bottom panels). The quality of fits are presented for $\mathrm{He}_{\mathrm{I}} \lambda 4388$ and He II $\lambda 4541$ lines (left column), and He I $\lambda 4471$ and He II $\lambda 4686$ lines (right column).

for the He I and He II lines for both components are shown in Fig. 1.

The reported values for the effective temperature of the primary of CW Cep have a broad range of almost $3000 \mathrm{~K}$, from $T_{\text {eff,p }}=28000 \pm 1000 \mathrm{~K}$ in Clausen \& Gimenez (1991) to a lower extreme of $T_{\text {eff,p }}=25400$ in Terrell (1991), and with intermediate values $T_{\text {eff,p }}=26500 \mathrm{~K}$ in Han et al. (2002) (Terrell 1991 and Han et al. 2002 fix $T_{\text {eff,p }}$ and do not report formal uncertainties for these values). It should be noted, however, that Terrell (1991) adopt their value for the primary effective temperature from a spectral type classification of $\mathrm{B} 0.5$, and Clausen \& Gimenez (1991) determine a mean value from different colour-calibrated photometric relations. In these studies, the effective temperature of the secondary $T_{\text {eff,s }}$ was then determined from the light curve solution. The reported spread in secondary effective temperature is only $1300 \mathrm{~K}$, with the hottest solution being only $600 \mathrm{~K}$ cooler than the primary (Clausen \& Gimenez 1991 ) and the coolest solution being $1900 \mathrm{~K}$ cooler than the primary (Terrell 1991). If we compare the values of our spectroscopically determined effective temperatures for the components of CW Cep, and the difference of their optimal values, $T_{\text {eff }, \mathrm{p}}=28300 \pm 460 \mathrm{~K}$ and $\Delta T_{\text {eff }}=750 \pm 620 \mathrm{~K}$, to the various estimates in previous analyses, we find the closest agreement with the estimates of Clausen \& Gimenez (1991). 
Table 3. Abundances determined for the components of binary system CW Cep.

\begin{tabular}{lccccccc}
\hline \hline Star & $\mathrm{C}$ & $\mathrm{N}$ & $\mathrm{O}$ & {$[\mathrm{N} / \mathrm{C}]$} & {$[\mathrm{N} / \mathrm{O}]$} & $\mathrm{Mg}$ & $\mathrm{Si}$ \\
\hline CW Cep A & $8.30 \pm 0.07$ & $7.79 \pm 0.08$ & $8.71 \pm 0.07$ & $-0.51 \pm 0.11$ & $-0.92 \pm 0.11$ & $7.55 \pm 0.08$ & $7.49 \pm 0.06$ \\
CW Cep B & $8.24 \pm 0.07$ & $7.70 \pm 0.08$ & $8.70 \pm 0.06$ & $-0.54 \pm 0.11$ & $-1.00 \pm 0.10$ & $7.53 \pm 0.09$ & $7.45 \pm 0.07$ \\
\hline OB binaries & $8.26 \pm 0.05$ & $7.70 \pm 0.04$ & $8.71 \pm 0.04$ & $-0.56 \pm 0.06$ & $-1.01 \pm 0.06$ & $7.59 \pm 0.08$ & $7.57 \pm 0.10$ \\
B single stars & $8.33 \pm 0.04$ & $7.79 \pm 0.04$ & $8.76 \pm 0.05$ & $-0.54 \pm 0.06$ & $-0.97 \pm 0.06$ & $7.56 \pm 0.05$ & $7.50 \pm 0.05$ \\
\hline
\end{tabular}

Notes. The atmospheric parameters used for the calculation of model atmospheres are given in Table 2. For the comparison the mean abundances for a sample of OB binaries given in Pavlovski et al. (2018), and for "present-day cosmic standard" determined for a sample of a single sharp-lined B-type stars in Nieva \& Przybilla (2012) are also presented.

Comparatively, we are able to reduce the uncertainties considerably due to our methodology combining the spectral disentangling, ionisation balancing, and fixing the surface gravity.

Following our atmospheric analysis, we also determine a detailed photospheric composition for the two stars. We calculate ATLAS9 model atmospheres for the atmospheric parameters derived above, from which theoretical spectra are calculated with the DETAIL/SURFACE suite. Details on the model atoms used can be found in Pavlovski et al. (2018). The abundances are then varied and optimised against the disentangled spectra, from which we report abundances for carbon, nitrogen, oxygen, magnesium, and silicon, as listed in Table 3. Additionally, we are able to derive the microturbulence velocity $\xi_{t}$ from the oxygen lines and the condition of null-dependence of the oxygen abundance on equivalent width. The derived $\xi_{\mathrm{t}}$ values for CW Cep A and B are listed in Table 2. For comparison, the present-day cosmic standard abundance pattern for sharp-lined early-B type stars of Nieva \& Przybilla (2012) is provided in the bottom row of Table 3, with which we find general agreement. We also note that the abundances of CW Cep are in close agreement with the abundance pattern and ratios derived for $\mathrm{OB}$ binaries by Pavlovski et al. (2018) as listed in the third row of Table 3.

Since iron lines are not visible in early B-type stars, the iron abundance cannot be directly measured and used as a proxy for stellar metallicity. Instead, Lyubimkov et al. (2005) determined the magnesium abundance from the $\mathrm{Mg}$ II line in a sample of 52 un-evolved early to mid-B-type stars and used this as a proxy for stellar metallicity. Lyubimkov et al. (2005) determined the mean abundance $\log \epsilon(\mathrm{Mg})=7.59 \pm 0.15$ to be in close agreement with the solar magnesium abundance, $\log \epsilon_{\odot}(\mathrm{Mg})=7.55 \pm 0.02$ as determined in Asplund et al. (2009). Exploiting the Mg abundance as a proxy for metallicity, Lyubimkov et al. (2005) find that the metallicty of young MS B-type stars in the solar neighbourhood and the Sun are the same. Following this work, we find that our reported magnesium abundance suggests that CW Cep has solar metallicity.

Additionally, we note that we observe $\mathrm{H} \alpha$ to be in emission in the new spectra assembled for this work. Figure 2 displays spectra at roughly quarter phases as labelled, all of which show clear double-peaked emission with central absorption. The corresponding velocity difference between the blue $(V)$ and red $(R)$ peaks remains constant at $\sim 105 \mathrm{~km} \mathrm{~s}^{-1}$ through the orbital phase. Similarly, we find that the intensity ration between the two peaks remains roughly stable at $V / R \sim 0.95$ throughout the orbit as well. For comparison, in Fig. 2 we also show synthetic $\mathrm{H} \alpha$ profile for the 0.25 phase. Although $\mathrm{H} \alpha$ emission is typical of Be stars or mass-transfer binaries, we cannot reliably attribute the emission to a given component. Moreover, as there is no clear evidence of variability of the emission with the orbital phase, we postulate that the emission originates from a

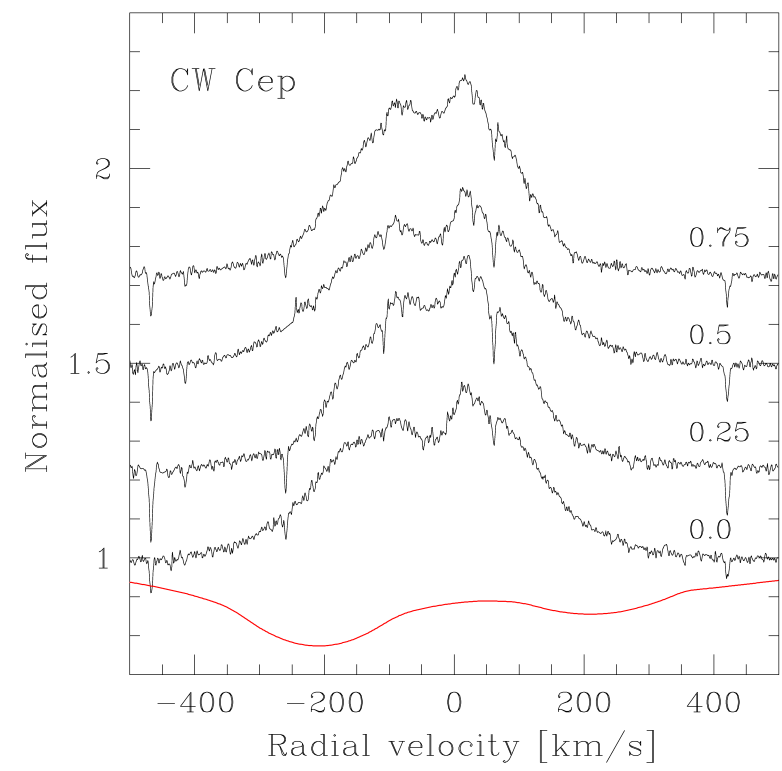

Fig. 2. Selected spectra corresponding to quarter phases centred around $\mathrm{H} \alpha$ showing constant emission throughout the orbit. A synthetic composite spectrum of CW Cep at quarter phase is shown in red for comparison. The radial velocity is calculated in the rest frame of the system.

circumbinary envelope or the nebula of the Cep OB3 association in which CW Cep is located. An extensive Hi nebula in which the Cep OB3 association is embeded is well documented (e.g. Simonson \& van Someren Greve 1976).

\section{2. $U O p h$}

U Oph consists of two main-sequence components of spectral type (mid-)B. Given that our NLTE grid discussed in the previous section is limited to stars hotter than $15000 \mathrm{~K}$, and that the use of the LTE formalism is justified for unevolved stars in this temperature range, we employ the Grid Search in Stellar Parameters (GSSP, Tkachenko 2015) code for the analysis of the disentangled spectra of the U Oph stellar components. The GSSP algorithm is based on a grid search in basic atmospheric parameters $\left(T_{\mathrm{eff}}, \log g, \xi, v \sin i\right.$, and $\left.[\mathrm{M} / \mathrm{H}]\right)$, and if necessary individual atmospheric abundances, and utilises a $\chi^{2}$ merit function and statistics to judge the goodness of fit between the grid of synthetic spectra and the observed spectrum and to compute $1 \sigma$ confidence intervals. Synthetic spectra are computed by means of the SYNTHV radiative transfer code (Tsymbal 1996) based on the pre-computed grid of LLMODELS atmosphere models (Shulyak et al. 2004). The atmosphere models and the synthetic spectra can be computed for arbitrary chemical compositions, where one, several, or all abundance values of the 
chemical elements can be set, also with an option of a vertical stratification in the stellar atmosphere. Similarly, the effect of the microturbulent velocity can be taken into account, if necessary assuming its vertical stratification.

The GSSP code is a multi-function software for spectrum analysis that is able to deal with spectra of single stars (GSSP_SINGLE module) and those of spectroscopic doublelined binaries, either with their observed composite spectra (GSSP_composite module) or with the disentangled spectra of individual stellar components (GSSP_SINGLE or GSSP_BINARY module). In the first of the two binary cases (GSSP_composite module), a composite spectrum of a binary is fitted with a grid of composite synthetic spectra that are built from all possible combinations of grid points for the primary and secondary star. Individual radial velocities can also be optimised along with all the above-mentioned atmospheric parameters of the two stars, where individual flux contributions are taken into account by means of the stellar radii ratio factor. In the second case, the distinction is made whether the spectra are analysed as those of a single star with a certain light dilution factor (GSSP_SINGLE module, known as unconstrained fitting where the light dilution factor is assumed to be independent of wavelength) or if they are fitted simultaneously by optimising radii ratio to account for individual light contributions (GSSP_BINARY module, known as constrained fitting with wavelength dependence of individual light contributions taken into account). A simultaneous fit of the two disentangled spectra is essential when a binary consists of two stars that are significantly different from each other in terms of their atmospheric properties. In this instance, their relative light contributions will strongly depend on wavelength. In the instance where the two stars have similar atmospheric parameters, independent fitting of the disentangled spectra is justified, while still enforcing that the two wavelength-independent light factors ultimately add up to unity (see Tkachenko 2015, for a detailed discussion).

As with CW Cep, the atmospheric parameters of U Oph A and $\mathrm{B}$ are similar enough that we fit the disentangled spectra individually. Again, we use an iterative approach where the light ratios are first determined from the disentangled spectra, then used as priors in the light curve solution. The photometric surface gravities are then fixed and the light ratios are re-optimised along with the other atmospheric parameters from the disentangled spectra. We found the light factors to be $0.575 \pm 0.007$ and $0.425 \pm 0.008$. The final solution is presented in Table 2 , while the quality of the fit is demonstrated in Fig. 3.

\section{Revised photometric models}

Both CW Cep and U Oph have been studied extensively in the literature for several decades, with a heavy focus on the rapid apsidal motion displayed by the systems (Holmgren et al. 1991; Clausen \& Gimenez 1991; Han et al. 2002; Wolf et al. 2002, 2006; Erdem et al. 2004; Vaz et al. 2007; Budding et al. 2009). This study uses the updated mass ratio, semi-major axis, and effective temperatures of the primary and secondary obtained in Sects. 3 and 4 to determine updated dynamical masses, radii, and surface gravities from photometric modelling with PHOEBE (Prša \& Zwitter 2005; Prsa et al. 2011).

\subsection{Photometric data}

For CW Cep we revisit the photometry initially analysed by Clausen \& Gimenez (1991). These data consist of 21 nights of observations spanning three years in the Stromgren uvby photometric system, totalling 1396 measurements in the uby filters,
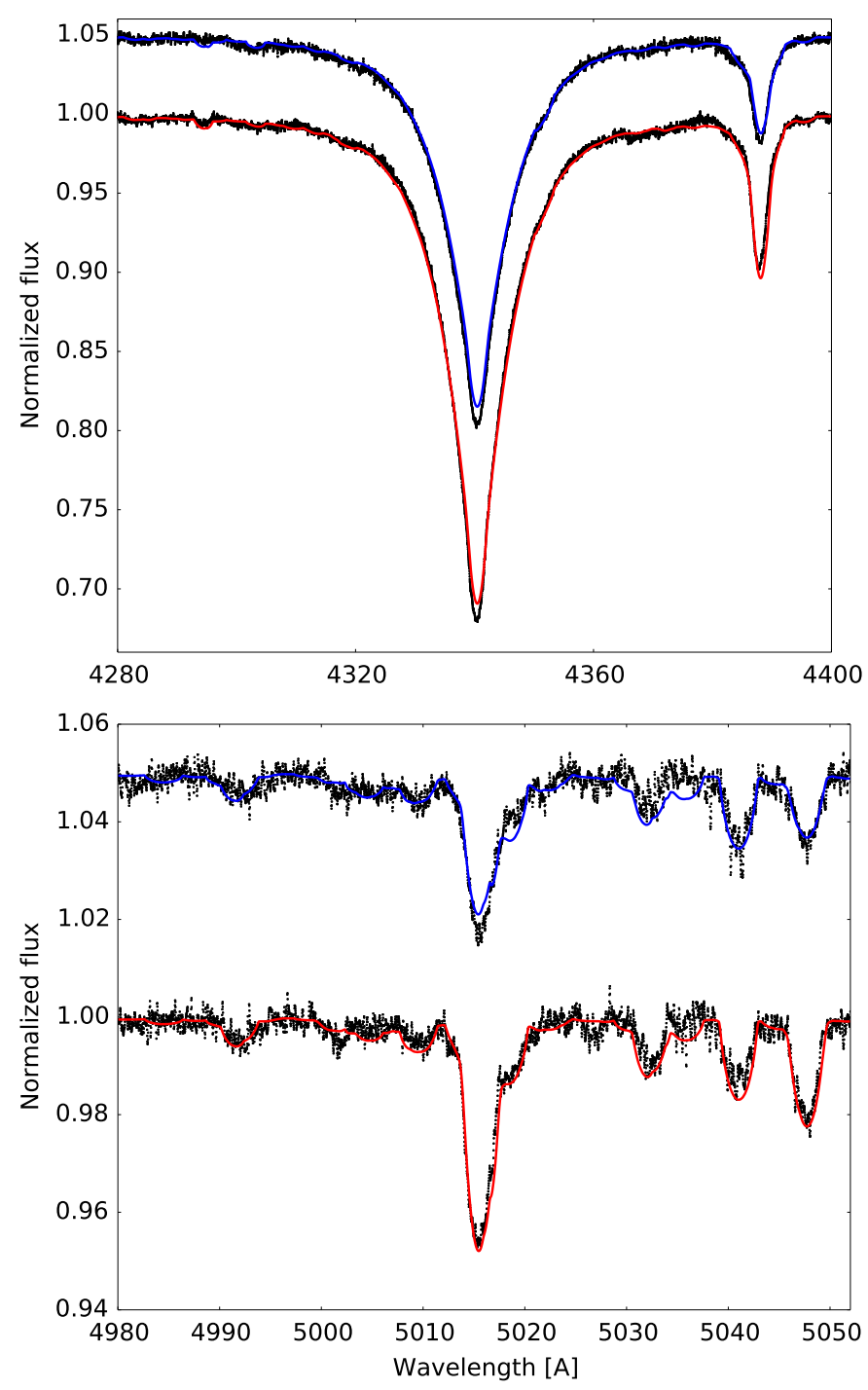

Fig. 3. Quality of the fit of the disentangled spectra (black dots) with the synthetic spectra computed from the best fit parameters listed in Table 2 (red and blue solid line for the primary and secondary component, respectively). The spectra of the secondary component were vertically shifted by a constant factor for clarity.

and 1318 in the $v$ filter. Both HD 218342 and HD 217035 served as photometric comparison stars, from which the final differential magnitudes were obtained. Extinction corrections determined by nightly coefficients calculated across the listed comparison stars and other standard objects were applied to the data (Gimenez et al. 1990). According to Clausen \& Gimenez (1991) the observations were constant to 0.004 mag in all filters, which we adopt as the uncertainty on each point.

We also revisit archival data for $\mathrm{U}$ Oph, initially analysed by Vaz et al. (2007). These data consist of 25 nights of observations spanning 1992-1994 in the Stromgren $u v b y$ photometric system, totalling 645 measurements; however, due to a trend in the data, we do not use the $u$-band light curve. The data were taken with the $0.5 \mathrm{~m}$ ESO SAT telescope in La Sille, Chile. HR 6367, HR 6353, and SAO 122251 were all used as comparison stars, from which the final differential magnitudes were obtained. As with CW Cep, extinction corrections were calculated each night from the comparison stars used. For more information on the comparison targets and observations, we refer 
to Vaz et al. (2007). Finally, Vaz et al. (2007) report a standard deviation of $0.0037 \mathrm{mag}$ in the $v b y$ filters, which we adopt as the uncertainty on each point.

\subsection{Photometric modelling methodology}

Both CW Cep and U Oph are well detached systems, exhibiting mild out-of-eclipse variability and slow apsidal motion, which for the purposes of our modelling is effectively mitigated by phase-binning the data. Our photometric modelling uses the PHOEBE binary modelling code, which is a modern extension of the original WD code but also incorporates new physics such as dynamic effects, the light travel time effect, and the reflection effect (Prša \& Zwitter 2005; Prsa et al. 2011). Given that all components considered are expected to have radiative envelopes, we fix the gravity darkening exponent to unity for all components (von Zeipel 1924). To obtain statistically robust estimates for the fit parameters, we wrap PHOEBE into a Bayesian Markov chain Monte Carlo (MCMC) framework using the EMCEE affine-invariant ensemble sampler MCMC code (Foreman-Mackey et al. 2013), which has already been successfully applied by Schmid et al. (2015), Hambleton et al. (2016), Pablo et al. (2017), Johnston et al. (2017), and Kochukhov et al. (2018).

MCMC procedures numerically evaluate Bayes' theorem, given by

$p(\Theta \mid d) \propto \mathcal{L}(d \mid \Theta) p(\Theta)$,

to estimate the posterior probability $p(\Theta \mid d)$ of some varied parameters $\Theta$ given the data $d$.We can see above that $p(\Theta \mid d)$ is proportional to the product of the likelihood function $\mathcal{L}(\Theta \mid d)$ and the prior probability of the parameter vector $p(\Theta)$. We write the likelihood function as

$\ln \mathcal{L} \propto-\frac{1}{2} \sum_{i}\left(\frac{d_{i}-y(\Theta)_{i}}{\sigma_{i}}\right)^{2}$,

where $y(\Theta)_{i}$ is each individual model point and $\sigma_{i}$ are the individual uncertainties associated with the data. We use the loglikelihood function as written here as this is what is used in practice. To make efficient use of the information obtained via the spectroscopic analysis, we apply Gaussian priors on the light factors (per cent of contribution per component) and $v \sin i$ estimates per component, as well as the projected binary separation $a \sin i$, the mass ratio $q$, the effective temperature of the secondary $T_{\text {eff, }, 2}$, and the eccentricity of the orbit. However, since PHOEBE does not directly sample all of them, we calculate the $v \sin i$ separately for each component and $a \sin i$ for every $\Theta$ considered. By including the spectroscopic light factors and simultaneously fitting all filters, we arrive at a more robust solution than if we were to fit them all individually and mitigate any degeneracies between the temperatures, light factors, and potentials of each component (Clausen \& Gimenez 1991). Furthermore, inclusion of priors on $v \sin i$ for each component helps constrain the spin paramters $f_{1}=\omega_{\text {rot }, 1} / \omega_{\text {orb }}$ and $f_{2}=\omega_{\text {rot }, 2} / \omega_{\text {orb }}$, which are otherwise largely unconstrained.

We draw parameter estimates and uncertainties as the median and $68.27 \%(1 \sigma)$ highest posterior density (HPD) intervals of the marginalised posterior distribution for each sampled parameter. As both systems undergo apsidal motion, we bin each light curve such that each phase bin covers 0.0033 phase units, which covers the entire periastron advance in a single binned point for either system. Although PHOEBE accepts $e$ and $\omega$ directly, we sample $e \sin \omega$ and $e \cos \omega$ in our MCMC analysis and solve for $e$ and $\omega$ afterwards. To aid in the discussion and provide additional constraints, we also report the relative radii in the bottom panel of Table 4.

\subsection{PHOEBE model: CW Cep}

To propagate our newly derived spectroscopic and orbital information into updated dynamical masses and radii, we fix the effective temperature of the primary $\left(\mathrm{T}_{\mathrm{eff}_{1}}\right)$ to the value listed in Table 2. As mentioned above, we apply Gaussian priors on the mass ratio, the eccentricity, the projected binary separation, the $v \sin i$ per component, and the light factor per component in the $v$-and $b$-band light curves since they correspond to the spectral range for which we derived the light factors. The light factor for the $u$ - and $y$-bands are given a uniform prior. For each sampled $\Theta$, we interpolate limb-darkening coefficients for the square-root law from the provided PHOEBE grids. Finally, given the radiative envelope of hot stars such as CW Cep A and B, we fix the albedo to unity in both components.

CW Cep is known to suffer from third light, which scales the apparent eclipse depths across each filter. Accounting for this scaling is non-trivial as there is a degeneracy between inclination and third light levels. However, it is crucial to account for this degeneracy when determining the derived masses. We use a uniform prior on the third light contributions per filter. Additionally, we sample the reference date $\left(\mathrm{HJD}_{0}\right)$, period $\left(P_{\text {orb }}\right)$, the inclination, the total binary separation, the secondary effective temperature, as well as potentials and synchronicity parameters per component $\left(\Omega_{1,2}\right.$ and $f_{1,2}$, respectively), giving all uniform priors. All sampled values, and the types of prior, are noted in Table 4.

The analysis of Clausen \& Gimenez (1991) states that the argument of periastron changes $24^{\circ}$ from $\sim 287^{\circ}$ to $\sim 311^{\circ}$ over the course of the photometric campaign, which corresponds to the secondary minima shifting $\sim 0.007$ phase units. To mitigate this change in periastron, we phase bin our data to 300 points, with each bin covering 0.0033 phase. Thus, the argument of periastron that we sample does not correspond to the value provided in the literature of the zero point, but rather to the mean periastron during the photometric campaign.

The third column of Table 4 shows the median and HPD estimates for the best fitting model. These values were used to construct the models seen in Fig. 4. For a consistency check, we compare the luminosities derived from the binary modelling with the luminosity derived from the Gaia parallax for CW Cep: $\pi_{\mathrm{G}}=1.04 \pm 0.49$ mas (Luri et al. 2018; Lindegren et al. 2018). We take $A_{v}=1.96$ following the reported value of $E(b-y)$ from Clausen \& Gimenez (1991) and $B C_{v}=2.95 \pm 0.05$ calculated as the average correction between CW Cep A and B (Reed 1998). The summed luminosity derived from our binary model yields $\log \frac{L}{L_{\odot}}=4.48 \pm 0.02$, while the Gaia derived luminosity yields $\log \frac{L_{\mathrm{G}}}{L_{\odot}}=4.78 \pm 0.41$. Given the large uncertainty $(\sim 50 \%)$ on the Gaia parallax, we also check the luminosity derived from the Hipparcos parallax $\left(\pi_{\mathrm{H}}=1.57 \pm 0.69\right.$ mas; van Leeuwen 2007), which yields $\log \frac{L_{\mathrm{H}}}{L_{\odot}}=4.42 \pm 0.4$. We find that all of these agree within the uncertainties.

\subsection{PHOEBE model: U Oph}

As with CW Cep, we fix the effective temperature of the primary to the value listed in Table 2 and impose Gaussian priors on $q, a \sin i, e$, and $v \sin i$ per component, and the light factors per 
Table 4. Binary model parameters for CW Cep and U Oph.

\begin{tabular}{|c|c|c|c|c|}
\hline \multirow[b]{2}{*}{ Parameter } & \multicolumn{2}{|c|}{ CW Cep } & \multicolumn{2}{|c|}{ U Oph } \\
\hline & Prior & HPD estimate & Prior & HPD estimate \\
\hline \multicolumn{5}{|c|}{ Sampled parameters } \\
\hline$L_{1, u}[\%]$ & $\mathcal{U}(40,70)$ & $57.3_{-1.0}^{+1.1}$ & - & - \\
\hline$L_{1, v}[\%]$ & $\mathcal{N}(56.5,0.5)$ & $56.7_{-1.0}^{+1.0}$ & $\mathcal{N}(57.5,0.7)$ & $57.2_{-5.2}^{+2.3}$ \\
\hline$L_{1, b}[\%]$ & $\mathcal{N}(56.5,0.5)$ & $56.6_{-1.0}^{+1.0}$ & $\mathcal{N}(57.5,0.7)$ & $57.1_{-5.1}^{+2.2}$ \\
\hline$L_{1, y}[\%]$ & $\mathcal{U}(40,70)$ & $56.5_{-1.0}^{+1.1}$ & $\mathcal{U}(40,70)$ & $57.0_{-5.2}^{+2.1}$ \\
\hline$L_{3, u}[\%]$ & $\mathcal{U}(0,15)$ & $0.6_{-0.5}^{+0.6}$ & - & - \\
\hline$L_{3, v}[\%]$ & $\mathcal{U}(0,15)$ & $1.9_{-0.5}^{+0.6}$ & $\mathcal{U}(0,15)$ & $0.8_{-0.3}^{+0.2}$ \\
\hline$L_{3, b}[\%]$ & $\mathcal{U}(0,15)$ & $2.8_{-0.5}^{+0.5}$ & $\mathcal{U}(0,15)$ & $1.1_{-0.2}^{+0.2}$ \\
\hline$L_{3, y}[\%]$ & $\mathcal{U}(0,15)$ & $3.6_{-0.4}^{+0.6}$ & $\mathcal{U}(0,15)$ & $1.3_{-0.2}^{+0.2}$ \\
\hline$T_{\mathrm{eff}, \mathrm{s}}[\mathrm{K}]$ & $\mathcal{N}(27550,600)$ & $27420_{-120}^{+150}$ & $\mathcal{N}(15620,200)$ & $15820_{-90}^{+90}$ \\
\hline$P_{\text {orb }}[\mathrm{d}]$ & $\mathcal{U}(1,5)$ & $2.7291316_{-3 e-7}^{+4 e-7}$ & $\mathcal{U}(1,4)$ & $1.67734590_{-2 e-8}^{+2 e-8}$ \\
\hline $\mathrm{HJD}_{0}[\mathrm{~d}]$ & $\mathcal{U}(-2,2)+2441669$ & $0.5831_{-0.0006}^{+0.0005}$ & $\mathcal{U}(-2,2)+2449161$ & $0.61101_{-0.00002}^{+0.0003}$ \\
\hline$i[\mathrm{deg}]$ & $\mathcal{U}(70,90)$ & $81.804_{-0.004}^{+0.006}$ & $\mathcal{U}(70,90)$ & $87.86_{-0.08}^{+0.1}$ \\
\hline$e \sin \omega_{0}$ & $\mathcal{U}(-0.0287,0.0287)$ & $-0.02544_{-2 e-5}^{+2 e-5}$ & $\mathcal{U}(-0.003,0.003)$ & $0.00189_{-1 e-5}^{+1 e-5}$ \\
\hline$e \cos \omega_{0}$ & $\mathcal{U}(-0.0287,0.0287)$ & $0.01329_{-3 e-5}^{+4 e-5}$ & $\mathcal{U}(-0.003,0.003)$ & $0.00233_{-1 e-5}^{+1 e-5}$ \\
\hline$a\left[R_{\odot}\right]$ & $\mathcal{U}(5,40)$ & $24.01_{-0.04}^{+0.04}$ & $\mathcal{U}(5,40)$ & $12.66_{-0.03}^{+0.03}$ \\
\hline$q=\frac{M_{2}}{M_{1}}$ & $\mathcal{N}(0.92,0.002)$ & $0.919_{-0.005}^{+0.005}$ & $\mathcal{N}(0.90,0.01)$ & $0.90_{-0.01}^{+0.01}$ \\
\hline$\Omega_{1}$ & $\mathcal{U}(4.5,9)$ & $5.39_{-0.03}^{+0.05}$ & $\mathcal{U}(4,9)$ & $4.64_{-0.02}^{+0.02}$ \\
\hline$\Omega_{2}$ & $\mathcal{U}(4.5,9)$ & $5.43_{-0.05}^{+0.03}$ & $\mathcal{U}(4,9)$ & $4.84_{-0.05}^{+0.02}$ \\
\hline$f_{1}$ & $\mathcal{U}(0.5,2)$ & $1.06_{-0.03}^{+0.03}$ & $\mathcal{U}(0.5,2)$ & $1.07_{-0.07}^{+0.07}$ \\
\hline$f_{2}$ & $\mathcal{U}(0.5,2)$ & $1.03_{-0.03}^{+0.03}$ & $\mathcal{U}(0.5,2)$ & $1.16_{-0.07}^{+0.08}$ \\
\hline \multicolumn{5}{|c|}{ Geometric parameters } \\
\hline$r_{1}$ & & $0.227_{-0.002}^{+0.001}$ & & $0.2715_{-0.0005}^{+0.0005}$ \\
\hline$r_{2}$ & & $0.212_{-0.001}^{+0.002}$ & & $0.2408_{-0.0009}^{+0.0007}$ \\
\hline
\end{tabular}

Notes. The top panel shows the parameters sampled during the MCMC run. For each parameter we list the units (when applicable), the priors, and the estimated values from the median and HPD confidence intervals. The bottom panel shows the derived geometric parameters and their estimates. Gaussian priors are listed with an $\mathcal{N}$, followed by their mean and width, and uniform priors are listed with a $\mathcal{U}$, followed by their boundaries.

component in the $v$ - and $b$-band light curves. Although we can safely ignore the small eccentricity and set it to zero to perform SPD, we cannot ignore the eccentricity in the light curve. As such, we apply a Gaussian prior according to the values taken from Vaz et al. (2007). Limb-darkening coefficients are interpolated from PHOEBE tables at every model evaluation. The albedos of both components are fixed to unity as both stars are expected to have radiative envelopes.

Since U Oph is also known to suffer from third light, we take the same approach as with CW Cep, using uniform priors for the third light per filter and uniform priors in all other parameters listed in Table 4. To mitigate the effects of the apsidal advance, we phase bin into 300 bins, which effectively covers the apparent change in superior - inferior conjunction. Again, this means that the argument of periastron reported is an average over the photometric campaign when the data was collected. The best model according to the median estimates listed in Table 4 is shown in Fig. 5. Derived parameters for both CW Cep and $\mathrm{U}$ Oph are reported in Table 5 alongside other solutions from the literature.

As with CW Cep, we compare the total luminosity obtained from binary modelling with the luminosities derived from Gaia $\left(\pi_{\mathrm{G}}=3.74 \pm 0.13\right.$ mas; Luri et al. 2018; Lindegren et al. 2018) and Hipparcos $\left(\pi_{H}=4.99 \pm 0.41\right.$ mas van Leeuwen 2007), assuming $A_{v}=0.72 \pm 0.2$, as taken from Vaz et al. (2007). The luminosity we calculate as $\log \frac{L}{L_{\odot}}=3.12 \pm 0.01$ does not agree with the Gaia derived luminosity as $\log \frac{L_{G}}{L_{\odot}}=3.27 \pm 0.09$ within $1 \sigma$, but does agree with the HipParcos derived luminosity as $\log \frac{L}{L_{\odot}}=3.02 \pm 0.1$ within $1 \sigma$.

\section{Evolutionary modelling}

\subsection{Evolutionary modelling set-up}

The updated masses, radii, and effective temperatures of CW Cep and U Oph provide strong discriminating power against stellar models. As discussed by Constantino \& Baraffe (2018) and Johnston et al. (2019), however, even such precision does not provide enough of a constraint to uniquely determine the extent of the near-core mixing region. We instead consider the convective core mass, and treat the near-core mixing, parameterised by a diffusive exponentially decaying overshooting prescription with a scaled extent $f_{\text {ov }}$ as a nuisance parameter. To do this, we fit each component to a grid of isochrone-clouds as described by Johnston et al. (2019). The isochrone-clouds are constructed from MESA tracks computed at solar metallicity $Z=0.014$, with the helium mass fraction fixed according to the Cosmic B-star standard $Y=0.276$ 


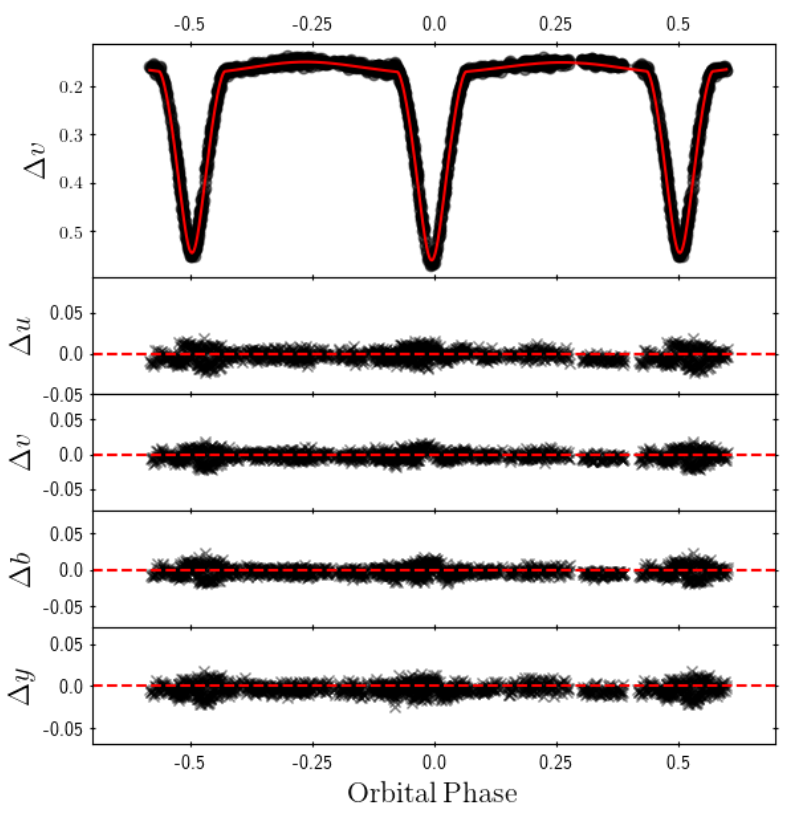

Fig. 4. Top panel: CW Cep PHOEBE model (solid red) for the Stromgren $v$ light curve (black $x$ 's) constructed from median values reported in Table 4. Bottom panels: residual light curves in the uvby filters after the best model has been removed. The dashed red line denotes the zero point to guide the eye.

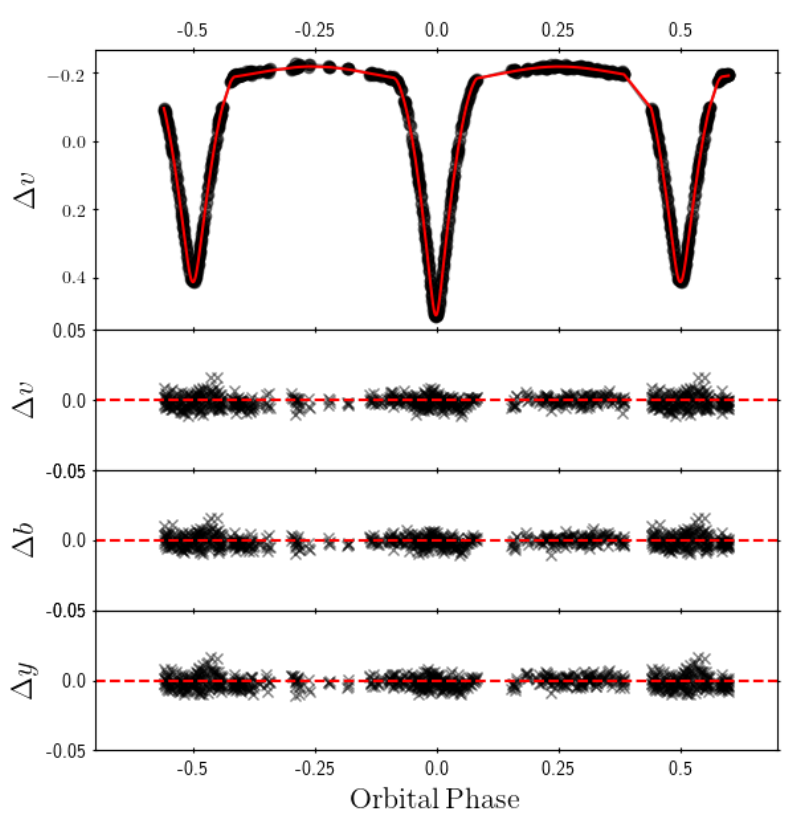

Fig. 5. Top panel: U Oph PHOEBE model (solid red) for the Stromgren $v$ light curve (black $x$ 's) constructed from median values reported in Table 4. Bottom panels: residual light curves in the $v$ by filters after the best model has been removed. The dashed red line denotes the zero point to guide the eye.

(Nieva \& Przybilla 2012), with $\alpha_{\text {MLT }}=1.8$ using MESA-r10398 (Paxton et al. 2011, 2018). We consider an isochrone-cloud at a given age $\tau$ to cover the range $f_{\mathrm{ov}} \in[0.005-0.04 ; 0.005]$ assuming the diffusive exponential description of overshooting implemented in MESA.

We adopt the Mahalanobis distance (MD) as our merit function, as applied in Johnston et al. (2019) and thoroughly discussed in Aerts et al. (2018). Using the MD as our merit function allows us to account for correlations present amongst model parameters that would otherwise compromise our solution (Aerts et al. 2018; Johnston et al. 2019). We choose to fit the mass, adopting the errors listed in Table 5 instead of interpolating the isochrone-clouds to the dynamical values. Since the $\mathrm{MD}$ is a maximum-likelihood point estimator, we perform Monte Carlo simulations (with 10000 iterations) to obtain confidence intervals on the model parameters and derived parameters of interest. We select the single best point returned for each iteration. By keeping only the best point, we sample the robustness of our solution given our grid. If we were to keep the best $N$ points, this would instead sample the variance of our solution space as a function of our grid and observables, and although this is an interesting phenomenon, it is ultimately not the focus of this work. After 10000 iterations, we bin the resulting distributions for all parameters of interest and apply 95\% HPD confidence intervals. The results are listed in Table 6.

\subsection{Modelling results and discussion}

The wide range of dynamical solutions for both CW Cep and U Oph shown in Table 5 gives us pause for thought. The spread between the minimum and maximum reported solutions is several times larger than the formal uncertainties reported, even though the same photometric data sets were used by different studies. The main difference across the individual solutions is the mass ratio, or more fundamentally the spectroscopic data sets. Furthermore, each set of radial velocities used to calculate the mass ratio was determined using different methods. Most critically, this translates into a large disparity in estimated ages for these systems, and therefore by necessity the estimated internal mixing. This is easily seen in the spread in ages for each system discussed earlier in Sect. 2. In addition to different masses and radii being used, different effective temperatures are also fit in the individual modelling efforts. In the end, these differences effectively mean that each study is modelling a different system. This highlights the need for a systematic evaluation of the accuracy versus the precision of dynamical and spectroscopic solutions for well-studied eclipsing binaries. However, this is beyond the scope of the present work. We note that future studies that entail modelling efforts of samples comprised of systems that were not homogeneously analysed must consider the systematic differences between different methods. We also note the necessity of allowing the mass ratio $q$ to vary. In the case where the mass ratio is fixed, the dynamical solution returns artificially high precision to the fourth decimal place or better. Given the high-precision échelle spectra, combined with stateof-the-art SPD and MCMC methodologies, we find our solution to be more robust than previous solutions, thus for the remainder of the discussion we only consider the results obtained in this work.

As discussed previously, the evolutionary modelling of eclipsing binaries involves several parameter degeneracies. While many studies attempt to constrain near-core mixing, the modelling procedure is not directly sensitive to the details of the prescriptions of these phenomena, but rather to their consequences. This means that any inference drawn on stellar rotation, convective overshooting, and/or magnetism from evolutionary modelling is convoluted with additional effects and uncertainties, at least some of which can be attributed to the implementation of such effects as diffusive processes in stellar structure and evolution codes, and although we have shown that CW Cep A and B and U Oph A and B are rotating at roughly a 
Table 5. Derived parameters CW Cep and U Oph.

\begin{tabular}{|c|c|c|c|c|c|}
\hline \multicolumn{6}{|l|}{ CW Cep } \\
\hline Parameter & Gimenez et al. (1987) & Clausen \& Gimenez (1991) & Han et al. (2002) ${ }^{(a)}$ & Han et al. (2002) ${ }^{(b)}$ & This Work \\
\hline$M_{1}\left[M_{\odot}\right]$ & $11.9 \pm 0.1$ & $11.82 \pm 0.14$ & 13.49 & 12.93 & $13.00_{-0.07}^{+0.07}$ \\
\hline$M_{2}\left[M_{\odot}\right]$ & $11.2 \pm 0.1$ & $11.09 \pm 0.14$ & 12.05 & 11.84 & $11.94_{-0.07}^{+0.08}$ \\
\hline$R_{1}\left[R_{\odot}\right]$ & $5.40 \pm 0.1$ & $5.48 \pm 0.12$ & 6.03 & 5.97 & $5.45_{-0.06}^{+0.03}$ \\
\hline$R_{2}\left[R_{\odot}\right]$ & $4.95 \pm 0.1$ & $4.99 \pm 0.12$ & 4.60 & 4.56 & $5.09_{-0.03}^{+0.06}$ \\
\hline $\log g_{1}[\mathrm{dex}]$ & $4.05 \pm 0.02$ & $4.03 \pm 0.02$ & 4.01 & 3.99 & $4.079_{-0.005}^{+0.010}$ \\
\hline $\log g_{2}[\mathrm{dex}]$ & $4.10 \pm 0.02$ & $4.09 \pm 0.02$ & 4.19 & 4.19 & $4.102_{-0.010}^{+0.005}$ \\
\hline \multicolumn{6}{|l|}{ U Oph } \\
\hline Parameter & Holmgren et al. (1991) & Vaz et al. (2007) & Budding et al. (2009) & This Work & \\
\hline$M_{1}\left[M_{\odot}\right]$ & $4.93 \pm 0.05$ & $5.273 \pm 0.091$ & $5.13 \pm 0.08$ & $5.09_{-0.05}^{+0.06}$ & \\
\hline$M_{2}\left[M_{\odot}\right]$ & $4.56 \pm 0.04$ & $4.783 \pm 0.072$ & $4.56 \pm 0.07$ & $4.58_{-0.05}^{+0.05}$ & \\
\hline$R_{1}\left[R_{\odot}\right]$ & $3.29 \pm 0.06$ & $3.483 \pm 0.020$ & $3.41 \pm 0.03$ & $3.44_{-0.01}^{-0.05}$ & \\
\hline$R_{2}\left[R_{\odot}\right]$ & $3.01 \pm 0.05$ & $3.109 \pm 0.034$ & $3.08 \pm 0.03$ & $3.05_{-0.01}^{+0.01}$ & \\
\hline $\log g_{1}[\mathrm{dex}]$ & $4.10 \pm 0.01$ & $4.068 \pm 0.010$ & $4.08 \pm 0.01$ & $4.073_{-0.004}^{-0.01}$ & \\
\hline $\log g_{2}[\mathrm{dex}]$ & $4.14 \pm 0.02$ & $4.128 \pm 0.012$ & $4.12 \pm 0.01$ & $\begin{array}{l}{ }^{-0.004} \\
4.131_{-0.004}^{+0.004}\end{array}$ & \\
\hline
\end{tabular}

Notes. Compares derived fundamental parameters from this work to previous studies of CW Cep (top) and U Oph (bottom). ${ }^{(a)}$ Solution derived using spectroscopic values obtained by Popper \& Hill (1991). ${ }^{(b)}$ Solution derived using spectroscopic values obtained by Stickland et al. (1992).

Table 6. Monte Carlo isochrone-cloud modelling 95\% confidence intervals for CW Cep and U Oph.

\begin{tabular}{lll}
\hline \hline Parameter & CW Cep & U Oph \\
\hline Age [Myr] & $7.0_{-1}^{+1}$ & $57.5_{-2.5}^{+5.0}$ \\
$f_{\text {ov, } 1}$ & $0.025_{-0.02}^{+0.015}$ & $0.025_{-0.015}^{+0.015}$ \\
$f_{\text {ov, } 2}$ & $0.030_{-0.02}^{+0.01}$ & $0.015_{-0.01}^{+0.015}$ \\
$M_{1}\left[M_{\odot}\right]$ & $13.00_{-0.16}^{+0.1}$ & $5.08_{-0.06}^{+0.01}$ \\
$M_{2}\left[M_{\odot}\right]$ & $12.00_{-0.11}^{+0.11}$ & $4.60_{-0.05}^{+0.05}$ \\
$X_{c, 1}$ & $0.54_{-0.03}^{+0.01}$ & $0.48_{-0.04}^{+0.02}$ \\
$X_{c, 2}$ & $0.57_{-0.03}^{+0.01}$ & $0.51_{-0.02}^{+0.03}$ \\
$M_{c c, 1}\left[M_{\odot}\right]$ & $4.34_{-0.29}^{+0.11}$ & $1.05_{-0.11}^{+0.08}$ \\
$M_{c c, 2}\left[M_{\odot}\right]$ & $3.86_{-0.19}^{+0.12}$ & $0.93_{-0.05}^{+0.06}$ \\
\hline
\end{tabular}

quarter of their critical rotation rates, any internal mixing caused by this will be degenerate, with mixing caused by convective overshooting. Therefore, reflecting this and the discussions presented by Constantino \& Baraffe (2018) and Johnston et al. (2019), we gear our discussion towards the core properties, to which evolutionary modelling is more directly sensitive since these properties dictate the stellar evolutionary sequence.

Despite the per-cent level precision on dynamical quantities provided by the binary solution, our modelling was not able to provide a constrained range for the extent of near-core mixing for the primary of $\mathrm{CW}$ Cep or for either component in U Oph. However, our modelling shows that CW Cep B requires a large amount of internal mixing to have its current observed properties and be co-evolutionary with CW Cep A. Stated differently, CW Cep B requires a more massive core than models based solely on the Schwarzchild criterion, otherwise it would appear to have a different age compared to CW Cep A. The left panels of Figs. 6 and 7 show how the isochrone-clouds of ages reported in Table 6 cover large and often overlapping regions of the spectroscopic parameter space due to the spread in near-core mixing. As can be seen in the right panels of these two figures, this translates to a generally more confined region in core properties, shown respectively as black circles and black x's for the primary and secondary for both systems. At the age and mass range
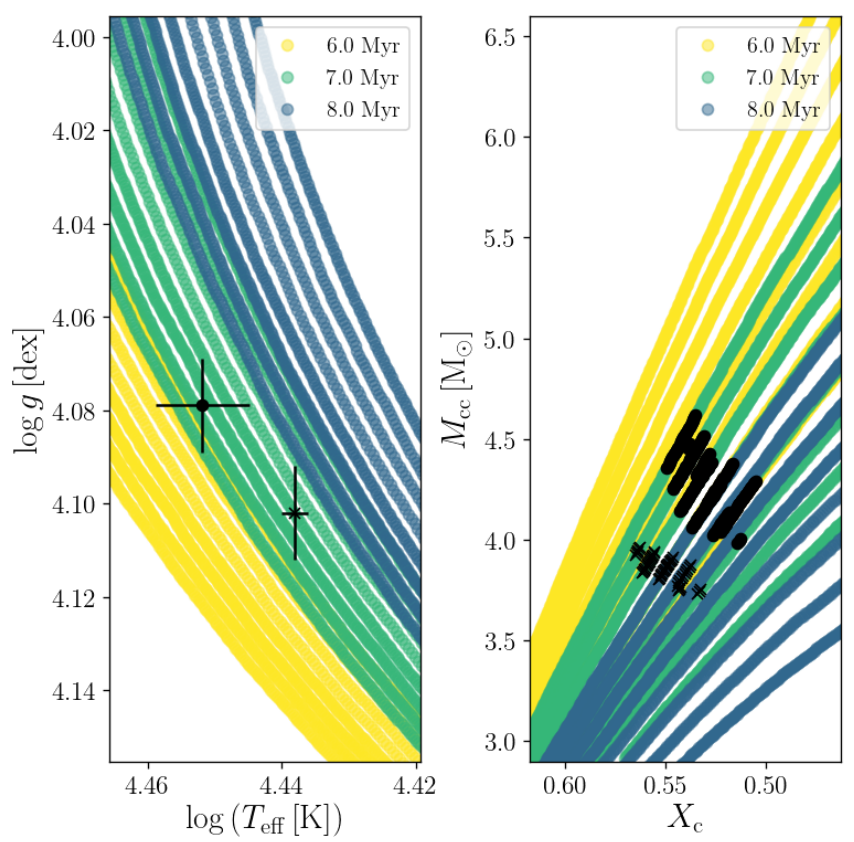

Fig. 6. Left: isochrone-clouds for the ages reported in Table 6 with the spectroscopic uncertainties plotted for CW Cep A and B in black. Right: convective mass plotted against the core hydrogen content for the isochrone-clouds shown in the left panel in grey. Those regions which are allowed by the spectroscopic uncertainties for CW Cep A and B are shown as black circles and $x$ 's, respectively.

for CW Cep, the components have not progressed sufficiently through their MS lifetimes to be able to critically constrain their core properties, with the primary and secondary being respectively $\sim 27 \%$ and $\sim 21 \%$ through their MS lifetimes. The cores of CW Cep A and B contain $\sim 33_{-4}^{+1} \%$ and $\sim 32_{-2}^{+1} \%$ of the total mass, respectively. For U Oph, the primary and secondary are $\sim 40 \%$ and $\sim 31 \%$ through their respective MS lifetimes. In this case, the resulting core parameter regions are more constrained. The cores of U Oph A and B contain $\sim 19_{-1}^{+3} \%$ and $\sim 19_{-0.5}^{+2} \%$ of the total mass, respectively. 

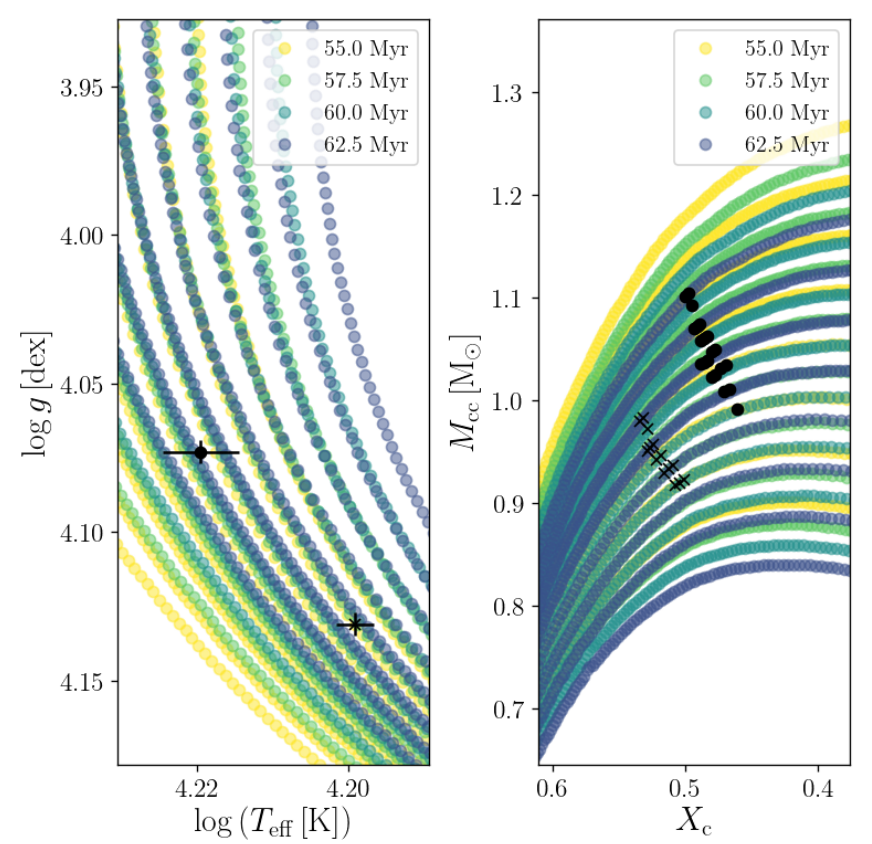

Fig. 7. Same as Fig. 6, but for U Oph.

The age estimate we find for CW Cep largely agrees with the estimates of Jordi et al. (1996) and Clausen \& Gimenez (1991), but is nearly twice as old as the solution reported by Ribas et al. (2000b). Our age estimate for U Oph is considerably higher than the median reported value in the literature, but it does agree with the upper limits of those solutions reported with a lower metallicity closer to the value we find and use in our modelling. We note that in both cases the estimated ages agree with those reported by previous studies. However, the solutions presented here are systematically older than those presented by Schneider et al. (2014) for both CW Cep and U Oph. Furthermore, our solution for CW Cep shows that the components are much less progressed along the MS ( 27\% and $\sim 21 \%)$ than those reported by Schneider et al. (2014) ( 35-40\% and $\sim 30-35 \%)$. This discrepancy is similar in the case of U Oph, with Schneider et al. (2014) reported U Oph A and B being 50\% and 40\% through the MS, respectively, compared to the $\sim 40 \%$ and $\sim 31 \%$ progress that we report. However, Schneider et al. (2014) use a solution that is $\sim 5 \%$ more massive compared to ours. This again highlights the need for a homogeneously analysed sample to draw inferences on trends in core properties and the physical processes that influence them.

\subsection{Rotation, synchronicity, and circularisation}

According to our MCMC estimates, CW Cep A and U Oph B are both rotating super-synchronously by $2 \sigma$, while CW Cep B and $\mathrm{U}$ Oph A are both rotating synchronously within the uncertainties. By combining the synchronicity parameters $f_{1}, 2$ and the derived parameters for each binary, we can calculate the unprojected rotational velocity of each component. We were able to perform this calculation with the projected rotation velocities obtained in Sect. 4; however, since we applied this information as a prior in our MCMC analysis, using the synchronicity parameters makes use of the same information. From this, we find that CW Cep A and B are rotating at $v_{\mathrm{A}}=107 \pm 3 \mathrm{~km} \mathrm{~s}^{-1}$ and $v_{\mathrm{B}}=97 \pm 3 \mathrm{~km} \mathrm{~s}^{-1}$, respectively, and $\mathrm{U}$ Oph $\mathrm{A}$ and $\mathrm{B}$ are rotating at $v_{\mathrm{A}}=111 \pm 7 \mathrm{~km} \mathrm{~s}^{-1}$ and $v_{\mathrm{B}}=107 \pm 6 \mathrm{~km} \mathrm{~s}^{-1}$.
We also calculate the critical rotation rates for each star using the parameters listed in Table 5. We find the critical rotation rates for CW Cep A and B to be $v_{\text {crit, } \mathrm{A}}=551 \pm 3 \mathrm{~km} \mathrm{~s}^{-1}$ and $v_{\text {crit, } \mathrm{B}}=$ $546 \pm 3 \mathrm{~km} \mathrm{~s}^{-1}$. This reveals that CW Cep A and B are rotating at $19.6 \pm 0.6 \%$ and $17.7 \pm 0.6 \%$ of their critical rates, respectively. For U Oph A and B, we find $v_{\text {crit, } \mathrm{A}}=434 \pm 3 \mathrm{~km} \mathrm{~s}^{-1}$ and $v_{\text {crit,B }}=$ $437 \pm 2 \mathrm{~km} \mathrm{~s}^{-1}$, thus revealing them to be rotating at $25.6 \pm 2 \%$ and $24.4 \pm 1 \%$ of their critical rates, respectively.

Tidal theory gives predictions of synchronisation and circularisation timescales for binary systems (Zahn 1975, 1977). Using the results of our modelling, we find $\tau_{\text {sync }}=0.606 \pm$ $0.002 \mathrm{Myr}$ and $\tau_{\text {circ }}=62.9 \pm 1 \mathrm{Myr}$ for $\mathrm{CW}$ Cep and $\tau_{\text {sync }}=$ $0.0879 \pm 0.0005 \mathrm{Myr}$ and $\tau_{\text {circ }}=4.63 \pm 0.02 \mathrm{Myr}$ for $\mathrm{U} \mathrm{Oph}$. The ages obtained in our isochrone-cloud modelling are an order of magnitude older than the theoretical synchronisation timescales for either CW Cep or U Oph. CW Cep is significantly younger than its theoretical circularisation timescale, and given the masses of the components, it will evolve beyond the MS before circularisation occurs. U Oph, however, is already significantly older than its circularisation timescale, but is still observed to be eccentric. This observation fits with the presence of a third body that likely sends the system through Kozai-Lidov cycles, as opposed to having a constantly decaying eccentricity.

\section{Conclusions}

Contemporary binary modelling techniques have the ability to provide per-cent level (or better) precision on fundamental stellar parameter estimates to be compared against evolutionary models. These parameter estimates have been used by numerous studies, including this one, to attempt to constrain poorly understood near-core mixing processes which cause deviations from nominal stellar evolution. However, no clear consensus exists in the literature as to whether this is possible.

In this work we obtained and analysed new spectroscopic observations on the intermediate- to high-mass binaries CW Cep and U Oph. Our analysis yielded an updated mass ratio to be used for light curve modelling, as well as the first abundance patterns for these systems. The abundance patterns were revealed to be roughly solar, which was exploited in the isochrone-cloud evolutionary modelling.

We performed light curve modelling using a Bayesian MCMC optimisation routine wrapped around the PHOEBE binary modelling code to obtain updated and highly precise mass and radius estimates. These estimates roughly agree with past studies, but the spread in reported solutions is much wider than the precision reported for any solution. This raises a concern in relation to the robustness of the accuracy of a solution versus its precision. To test the consistency of our solutions, we compared the luminosities from binary modelling with those calculated from Gaia parallaxes. Furthermore, given the close separation of the components in these systems, they are ideal candidates for investigating the influence of the inclusion of second-order physics such as Doppler boosting and the reflection effect on resulting modelled core properties. However, this analysis requires high-precision space photometry, which has yet to be assembled for these systems but will be done soon by the TESS mission (Ricker et al. 2015). Additionally, the high-precision orbital and dynamical solutions allowed us to investigate the rotation rates and tidal synchronisation and circularisation timescales for both systems.

Using our updated dynamical solution and temperatures, we performed isochrone-cloud modelling following the procedure as described by Johnston et al. (2019) to obtain estimates 
on model input parameters and on derived parameters such as the core properties. Our results reveal that, given model degeneracies, we cannot critically constrain the extent of near-core mixing. We do, however, constrain the core mass and hydrogen content for the two components of both CW Cep and U Oph, as these quantities directly dictate the current evolutionary status of a star. We compare our results to those of Schneider et al. (2014), who performed a similar analysis, but which assumed rotational mixing instead of exponentially decaying diffusive convetive overshooting in their evolutionary models. Combined with the alarming spread in reported dynamical solutions shown in Table 5, our comparison highlights the need for a homogeneously analysed sample to be able to make meaningful inference on internal physical processes such as convective overshooting, rotational and pulsational mixing, and magnetism (Aerts et al. 2014). Finally, we ask that future studies that perform evolutionary modelling should report the core masses of their solutions in addition to the overshooting extent or near-core rotation rate.

Acknowledgements. We thank the referee for the helpful comments that have improved the manuscript. We thank Dr. Dominic Bowman and Prof. Hugues Sana for the discussions pertaining to error determination. The research leading to these results has received funding from the European Research Council (ERC) under the European Union's Horizon 2020 research and innovation programme (grant agreement $\mathrm{N}^{\circ}$ 670519: MAMSIE; CJ and AT), from the Research Foundation Flanders (FWO) under grant agreement G0A2917N (BlackGEM; CJ), from the KU Leuven Research Council (grant C16/18/005: PARADISE; AT), from the Research Foundation Flanders (FWO) under grant agreement G0H5416N (ERC Runner Up Project; AT), and from the BELgian federal Science Policy Office (BELSPO) through PRODEX grant PLATO (AT). KP acknowledges financial support from the Croatian Science Foundation under grant IP-2014-09-8656 (STARDUST). The computational resources and services used in this work were provided by the VSC (Flemish Supercomputer Center), funded by the Research Foundation - Flanders (FWO) and the Flemish Government, department EWI. Based on observations made with the Mercator Telescope, operated on the island of La Palma by the Flemmish Community, at the Spanish Observatorio del Roque de los Muchachos of the Instituto de Astrofísica de Canarias.

\section{References}

Aerts, C., \& Harmanec, P. 2004, ASP Conf. Ser., 318, 325

Aerts, C., Molenberghs, G., Kenward, M. G., \& Neiner, C. 2014, ApJ, 781, 88 Aerts, C., Mathis, S., \& Rogers, T. 2019, ARA\&A, 57

Aerts, C., Molenberghs, G., Michielsen, M., et al. 2018, ApJS, 237, 15

Andersen, J., Nordstroem, B., \& Clausen, J. V. 1990, ApJ, 363, L33

Asplund, M., Grevesse, N., Sauval, A. J., \& Scott, P. 2009, ARA\&A, 47, 481

Beck, P. G., Mathis, S., Gallet, F., et al. 2018a, MNRAS, 479, L123

Beck, P. G., Kallinger, T., Pavlovski, K., et al. 2018b, A\&A, 612, A22

Blaauw, A. 1961, Bull. Astron. Inst. Neth., 15, 265

Blaauw, A., Hiltner, W. A., \& Johnson, H. L. 1959, ApJ, 130, 69

Bonanos, A. Z., Stanek, K. Z., Kudritzki, R. P., et al. 2006, ApJ, 652, 313

Briquet, M., Morel, T., Thoul, A., et al. 2007, MNRAS, 381, 1482

Brott, I., de Mink, S. E., Cantiello, M., et al. 2011a, A\&A, 530, A115

Brott, I., Evans, C. J., Hunter, I., et al. 2011b, A\&A, 530, A116

Budding, E., Inlek, G., \& Demircan, O. 2009, MNRAS, 393, 501

Butler, K., Mendoza, C., \& Zeippen, C. J. 1984, J. Phys. B At. Mol. Phys., 17, 2039

Charbonneau, P. 1995, ApJS, 101, 309

Claret, A. 2007, A\&A, 475, 1019

Claret, A., \& Torres, G. 2016, A\&A, 592, A15

Claret, A., \& Torres, G. 2017, ApJ, 849, 18

Claret, A., \& Torres, G. 2018, ApJ, 859, 100

Claret, A., \& Torres, G. 2019, ApJ, 876, 134

Clausen, J. V., \& Gimenez, A. 1991, A\&A, 241, 98

Clements, G. L., \& Neff, J. S. 1979, ApJS, 41, 1

Constantino, T., \& Baraffe, I. 2018, A\&A, 618, A177

De Cat, P., Aerts, C., De Ridder, J., et al. 2000, A\&A, 355, 1015

De Cat, P., De Ridder, J., Hensberge, H., \& Ilijic, S. 2004, ASP Conf. Ser., 318, 338

de Mink, S. E., Langer, N., Izzard, R. G., Sana, H., \& de Koter, A. 2013, ApJ, 764,166
Eaton, J. A., \& Ward, D. H. 1973, ApJ, 185, 921

Ekström, S., Georgy, C., Eggenberger, P., et al. 2012, A\&A, 537, A146

Ekström, S., Meynet, G., Georgy, C., \& Granada, A. 2018, Memorie della Societa Astronomica Italiana, 89, 50

Elias, II., N. M., Koch, R. H., \& Pfeiffer, R. J. 2008, A\&A, 489, 911

Erdem, A., Soydugan, E., Soydugan, F., et al. 2004, Astron. Nachr., 325, 336 Foreman-Mackey, D., Hogg, D. W., Lang, D., \& Goodman, J. 2013, PASP, 125, 306

Frieboes-Conde, H., \& Herczeg, T. 1973, A\&AS, 12, 1

Gallenne, A., Pietrzyński, G., Graczyk, D., et al. 2016, A\&A, 586, A35

Giddings, J. R. 1981, PhD Thesis, University of London

Gimenez, A., Kim, C.-H., \& Nha, I.-S. 1987, MNRAS, 224, 543

Gimenez, A., Rolland, A., Garcia, J. M., \& Clausen, J. V. 1990, A\&AS, 86, 259

Grunhut, J. H., Wade, G. A., Leutenegger, M., et al. 2013, MNRAS, 428, 1686

Guinan, E. F., Fitzpatrick, E. L., DeWarf, L. E., et al. 1998, ApJ, 509, L21

Guinan, E. F., Ribas, I., Fitzpatrick, E. L., et al. 2000, ApJ, 544, 409

Hadrava, P. 1995, A\&AS, 114, 393

Hambleton, K., Kurtz, D. W., Prša, A., et al. 2016, MNRAS, 463, 1199

Han, W., Kim, C.-H., Lee, W.-B., \& Koch, R. H. 2002, AJ, 123, 2724

Hensberge, H., \& Pavlovski, K. 2007, IAU Symp., 240, 136

Hensberge, H., Pavlovski, K., \& Verschueren, W. 2000, A\&A, 358, 553

Herrero, A., Kudritzki, R. P., Vilchez, J. M., et al. 1992, A\&A, 261, 209

Higl, J., \& Weiss, A. 2017, A\&A, 608, A62

Hirschi, R., den Hartogh, J., Cristini, A., Georgy, C., \& Pignatari, M. 2014, XIII Nuclei in the Cosmos (NIC XIII), 1

Holmgren, D. E., Hill, G., \& Fisher, W. 1991, A\&A, 248, 129

Huffer, C. M., \& Kopal, Z. 1951, ApJ, 114, 297

Ignace, R., Hole, K. T., Oskinova, L. M., \& Rotter, J. P. 2017, ApJ, 850, 82

Ilijic, S., Hensberge, H., Pavlovski, K., \& Freyhammer, L. M. 2004, ASP Conf. Ser., 318,111

Ivezić, Ž., Connelly, A. J., VanderPlas, J. T., \& Gray, A. 2014, Statistics, Data Mining, and Machine Learningin Astronomy (Princeton: Princeton University Press)

Johnston, C., Buysschaert, B., Tkachenko, A., Aerts, C., \& Neiner, C. 2017, MNRAS, 469, L118

Johnston, C., Tkachenko, A., Aerts, C., et al. 2019, MNRAS, 482, 1231

Jordi, C., Trullols, E., \& Galadi-Enriquez, D. 1996, A\&A, 312, 499

Kaemper, B. C. 1986, Ap\&SS, 120, 167

Kirkby-Kent, J. A., Maxted, P. F. L., Serenelli, A. M., et al. 2018, A\&A, 620, C5

Koch, R. H., \& Koegler, C. A. 1977, ApJ, 214, 423

Kochukhov, O., Johnston, C., Alecian, E., \& Wade, G. A. 2018, MNRAS, 478, 1749

Kolbas, V., Dervisoğlu, A., Pavlovski, K., \& Southworth, J. 2014, MNRAS, 444, 3118

Lindegren, L., Hernández, J., Bombrun, A., et al. 2018, A\&A, 616, A2

Luri, X., Brown, A. G. A., Sarro, L. M., et al. 2018, A\&A, 616, A9

Lyubimkov, L. S., Rostopchin, S. I., Rachkovskaya, T. M., Poklad, D. B., \& Lambert, D. L. 2005, MNRAS, 358, 193

Maeder, A. 2009, Physics, Formation and Evolution of Rotating Stars (Berlin, Heidelberg: Springer)

Moravveji, E., Aerts, C., Pápics, P. I., Triana, S. A., \& Vandoren, B. 2015, A\&A, 580, A27

Moravveji, E., Townsend, R. H. D., Aerts, C., \& Mathis, S. 2016, ApJ, 823, 130

Nha, I. S. 1975, AJ, 80, 232

Nieva, M.-F., \& Przybilla, N. 2012, A\&A, 539, A143

Pablo, H., Richardson, N. D., Moffat, A. F. J., et al. 2015, ApJ, 809, 134

Pablo, H., Richardson, N. D., Fuller, J., et al. 2017, MNRAS, 467, 2494

Panchatsaram, T. 1981, Bull. Astron. Soc. India, 9, 139

Pavlovski, K., \& Hensberge, H. 2005, A\&A, 439, 309

Pavlovski, K., \& Hensberge, H. 2010, ASP Conf. Ser., 435, 207

Pavlovski, K., \& Southworth, J. 2009, MNRAS, 394, 1519

Pavlovski, K., Tamajo, E., Koubský, P., et al. 2009, MNRAS, 400, 791

Pavlovski, K., Southworth, J., \& Tamajo, E. 2018, MNRAS, 481, 3129

Paxton, B., Bildsten, L., Dotter, A., et al. 2011, ApJS, 192, 3

Paxton, B., Schwab, J., Bauer, E. B., et al. 2018, ApJS, 234, 34

Pietrzyński, G., Graczyk, D., Gieren, W., et al. 2013, Nature, 495, 76

Pols, O. R., Tout, C. A., Schroder, K.-P., \& Eggleton, P. P. 1997, MNRAS, 289, 869

Popper, D. M. 1974, ApJ, 188, 559

Popper, D. M. 1980, ARA\&A, 18, 115

Popper, D. M., \& Hill, G. 1991, AJ, 101, 600

Prsa, A., Matijevic, G., Latkovic, O., Vilardell, F., \& Wils, P. 2011, Astrophysics Source Code Library [record ascl:1106.002]

Prša, A., \& Zwitter, T. 2005, ApJ, 628, 426

Raskin, G., van Winckel, H., Hensberge, H., et al. 2011, A\&A, 526, A69

Reed, B. C. 1998, JRASC, 92, 36

Ribas, I., Guinan, E. F., Fitzpatrick, E. L., et al. 2000a, ApJ, 528, 692

Ribas, I., Jordi, C., \& Giménez, Á. 2000b, MNRAS, 318, L55 
Ribas, I., Jordi, C., Torra, J., \& Giménez, Á. 2000c, MNRAS, 313, 99

Ribas, I., Jordi, C., Vilardell, F., et al. 2005, ApJ, 635, L37

Ricker, G. R., Winn, J. N., Vanderspek, R., et al. 2015, J. Astron. Telesc. Instrum. Syst., 1,014003

Roxburgh, I. W. 1978, A\&A, 65, 281

Roxburgh, I. W. 1992, A\&A, 266, 291

Schmid, V. S., \& Aerts, C. 2016, A\&A, 592, A116

Schmid, V. S., Tkachenko, A., Aerts, C., et al. 2015, A\&A, 584, A35

Schneider, F. R. N., Langer, N., de Koter, A., et al. 2014, A\&A, 570, A66

Schroder, K.-P., Pols, O. R., \& Eggleton, P. P. 1997, MNRAS, 285, 696

Shulyak, D., Tsymbal, V., Ryabchikova, T., Stütz, C., \& Weiss, W. W. 2004, A\&A, 428, 993

Simon, K. P., \& Sturm, E. 1994, A\&A, 281, 286

Simón-Díaz, S., Godart, M., Castro, N., et al. 2017, A\&A, 597, A22

Simonson, III., S. C., \& van Someren Greve, H. W. 1976, A\&A, 49, 343

Stancliffe, R. J., Fossati, L., Passy, J. C., \& Schneider, F. R. N. 2015, A\&A, 575, A117

Stickland, D. J., Koch, R. H., \& Pfeiffer, R. J. 1992, Observatory, 112, 277

Suchomska, K., Graczyk, D., Pietrzyński, G., et al. 2019, A\&A, 621, A93

Takata, J., Okazaki, A. T., Nagataki, S., et al. 2012, ApJ, 750, 70

Tamajo, E., Pavlovski, K., \& Southworth, J. 2011, A\&A, 526, A76

Terrell, D. 1991, MNRAS, 250, 209

Tkachenko, A. 2015, A\&A, 581, A129

Tkachenko, A., Degroote, P., Aerts, C., et al. 2014, MNRAS, 438, 3093
Torres, G., Andersen, J., \& Giménez, A. 2010, ARA\&A, 18, 67

Torres, G., Ruíz-Rodríguez, D., Badenas, M., et al. 2013, ApJ, 773, 40

Torres, G., Sandberg Lacy, C. H., Pavlovski, K., et al. 2014a, ApJ, 797, 31

Torres, G., Vaz, L. P. R., Sandberg Lacy, C. H., \& Claret, A. 2014b, AJ, 147, 36

Tsymbal, V. 1996, ASP Conf. Ser., 108, 198

Valle, G., Dell'Omodarme, M., Prada Moroni, P. G., \& Degl'Innocenti, S. 2016 , A\&A, 587, A16

Valle, G., Dell'Omodarme, M., Prada Moroni, P. G., \& Degl'Innocenti, S. 2017, A\&A, 600, A41

Valle, G., Dell'Omodarme, M., Prada Moroni, P. G., \& Degl'Innocenti, S. 2018, A\&A, 615, A62

van Leeuwen, F. 2007, A\&A, 474, 653

Van Reeth, T., Tkachenko, A., \& Aerts, C. 2016, A\&A, 593, A120

Vaz, L. P. R., Andersen, J., \& Claret, A. 2007, A\&A, 469, 285

von Zeipel, H. 1924, MNRAS, 84, 665

Wade, G. A., Smoker, J. V., Evans, C. J., et al. 2019, MNRAS, 483, 2581

Wolf, M., Harmanec, P., Diethelm, R., Hornoch, K., \& Eenens, P. 2002, A\&A, 383,533

Wolf, M., Kučáková, H., Kolasa, M., et al. 2006, A\&A, 456, 1077

Zahn, J. P. 1975, A\&A, 41, 329

Zahn, J. P. 1977, Penetrative Convection in Stars (Berlin and New York: Springer-Verlag), 225

Zahn, J. P. 1991, A\&A, 252, 179 


\section{Appendix A: CW Cep Marginalised posterior distributions}

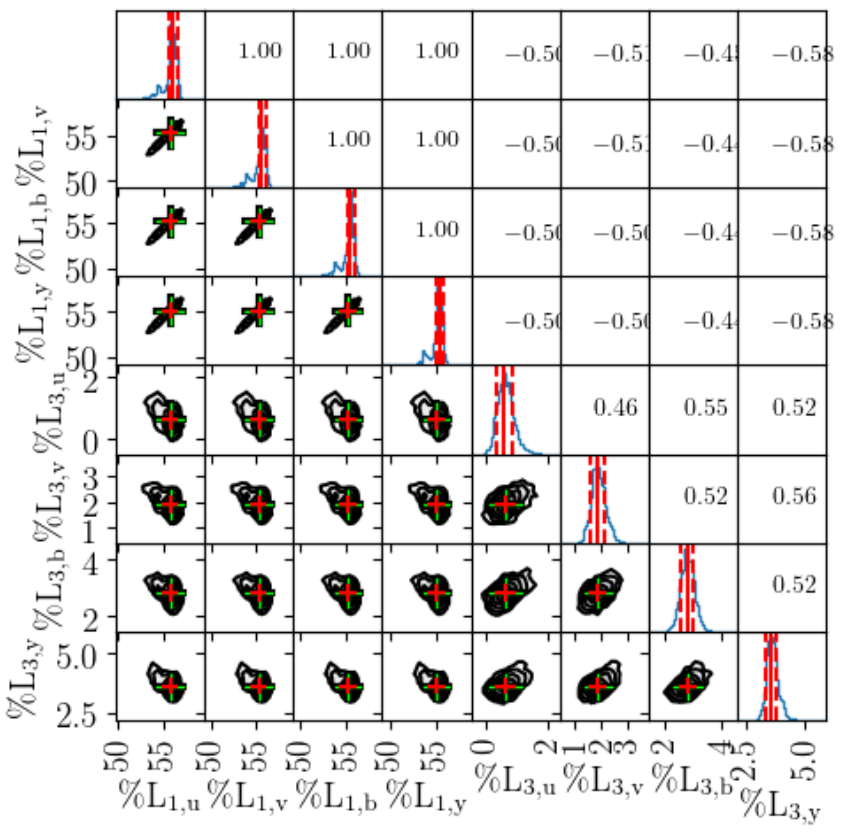

Fig. A.1. Marginalised posterior distributions for the primary and secondary passband luminosities for each observed filter. The median is shown by a solid vertical red line, the upper and lower bounds for $68.27 \%$ CI by dashed vertical red lines.

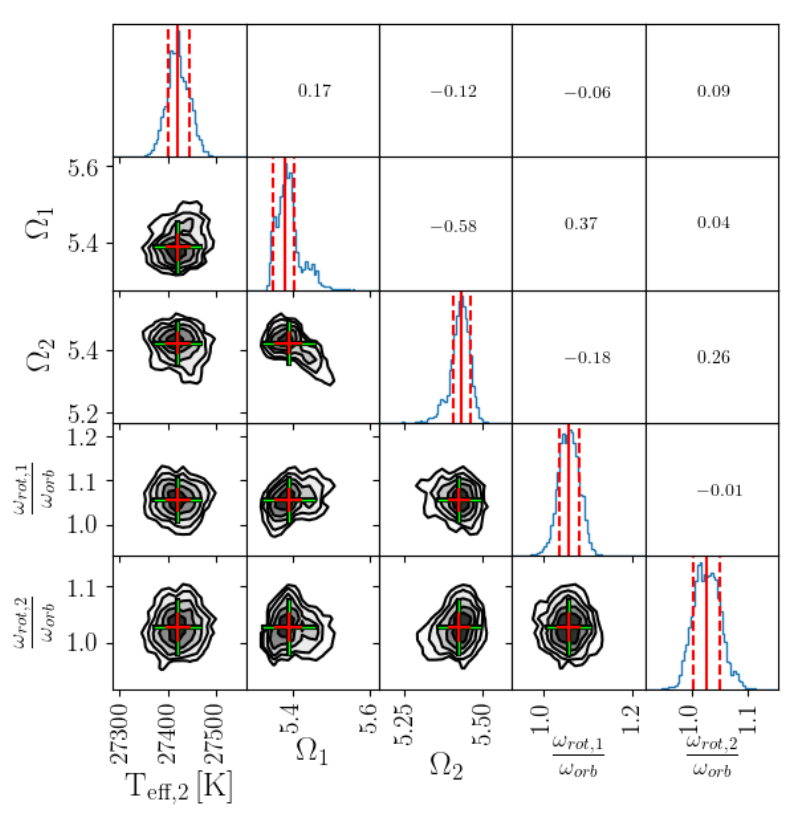

Fig. A.2. Marginalised posterior distributions for the primary and secondary parameters. The median is shown by a solid vertical red line, the upper and lower bounds for $68.27 \%$ CI by dashed vertical red lines.

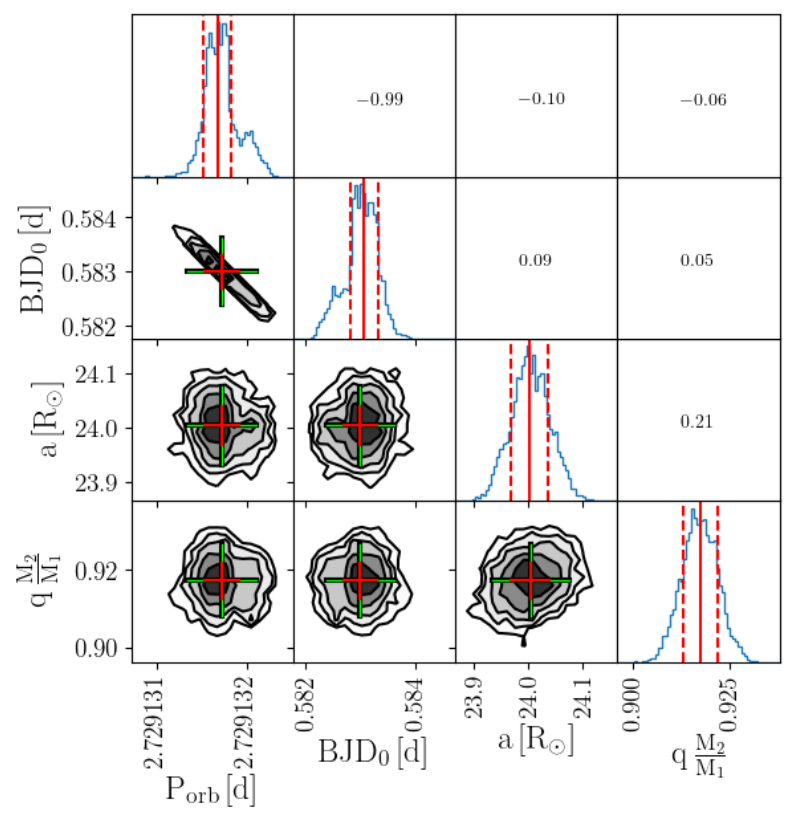

Fig. A.3. Marginalised posterior distributions for the orbital parameters. The median is shown by a solid vertical red line, the upper and lower bounds for $68.27 \%$ CI by dashed vertical red lines.

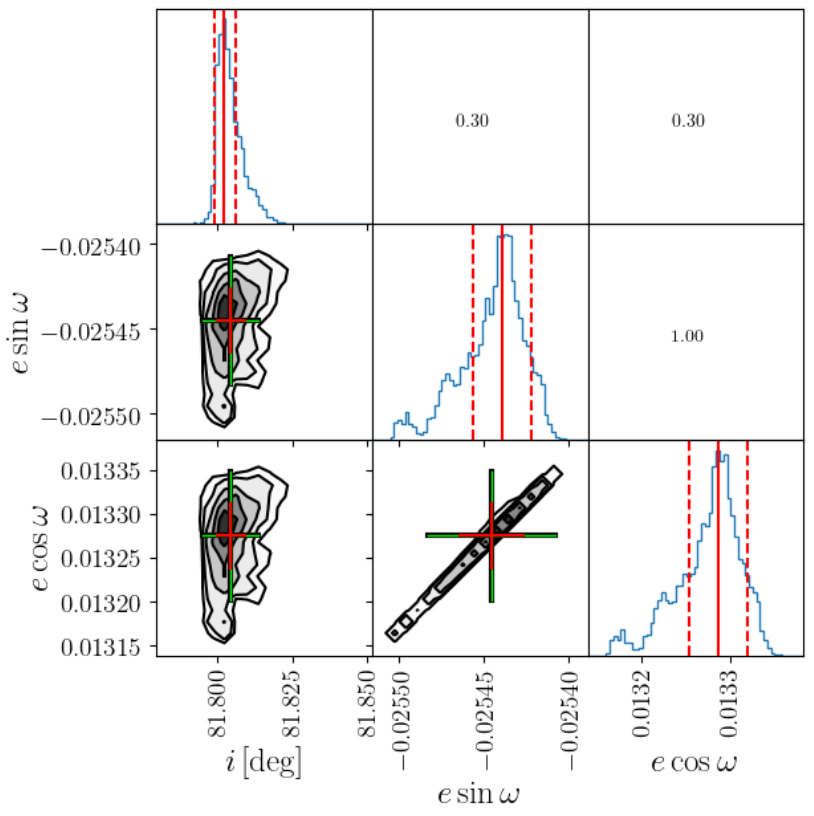

Fig. A.4. Marginalised posterior distributions for the system parameters. The median is shown by a solid vertical red line, the upper and lower bounds for $68.27 \%$ CI by dashed vertical red lines. 
Appendix B: U Oph Marginalised posterior distributions

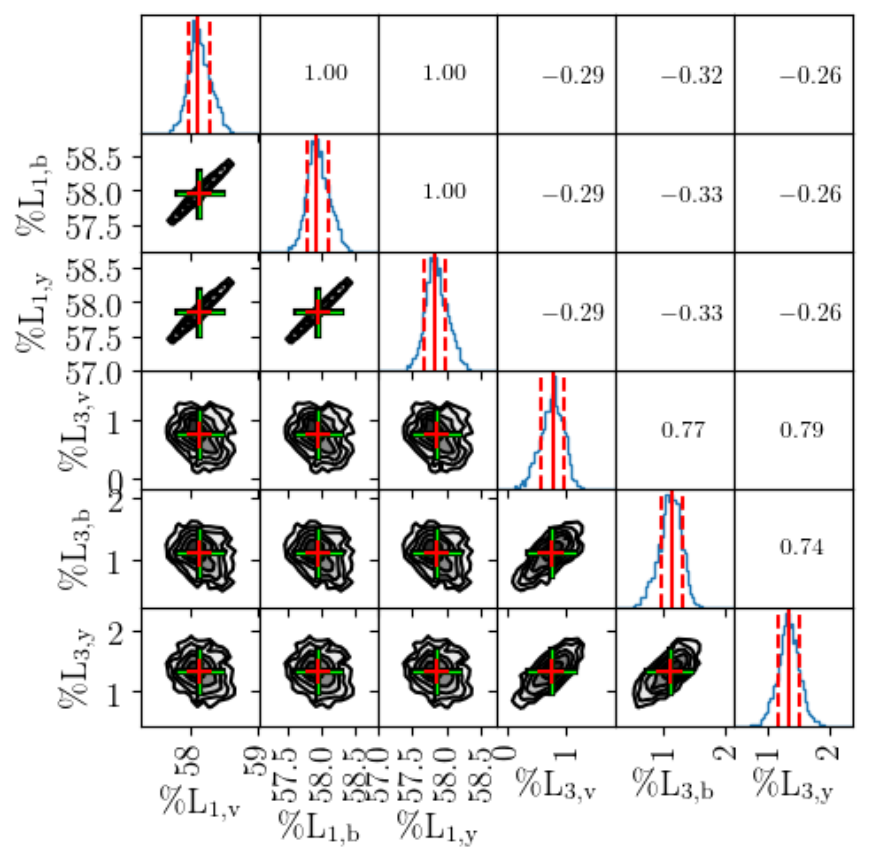

Fig. B.1. Marginalised posterior distributions for the primary and secondary passband luminosities for each observed filter. The median is shown by a solid vertical red line, the upper and lower bounds for $68.27 \%$ CI by dashed vertical red lines.

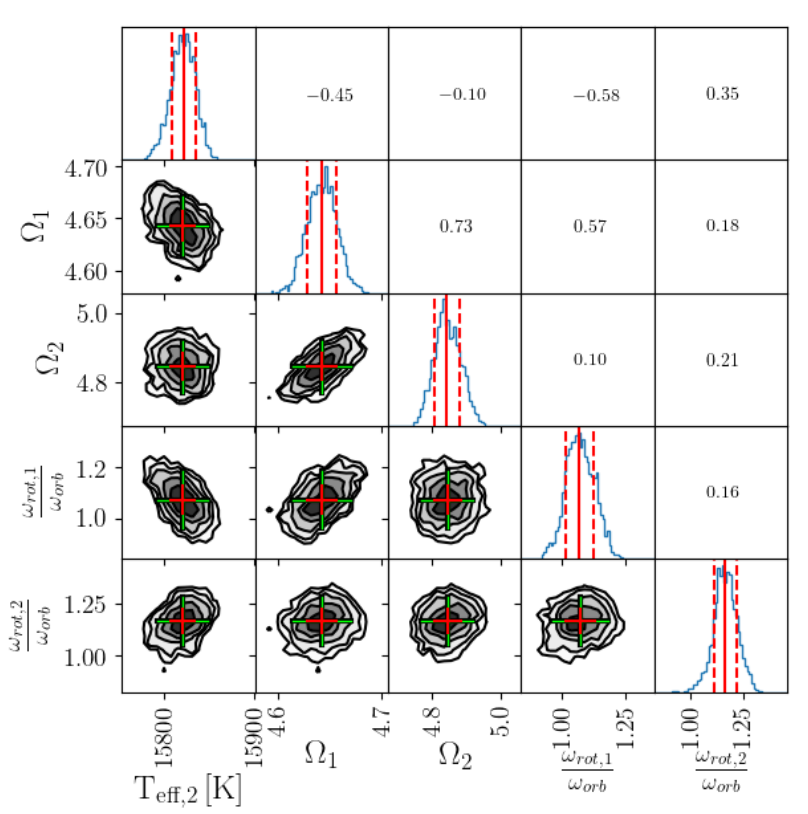

Fig. B.2. Marginalised posterior distributions for the primary and secondary parameters. The median is shown by a solid vertical red line, the upper and lower bounds for $68.27 \%$ CI by dashed vertical red lines.

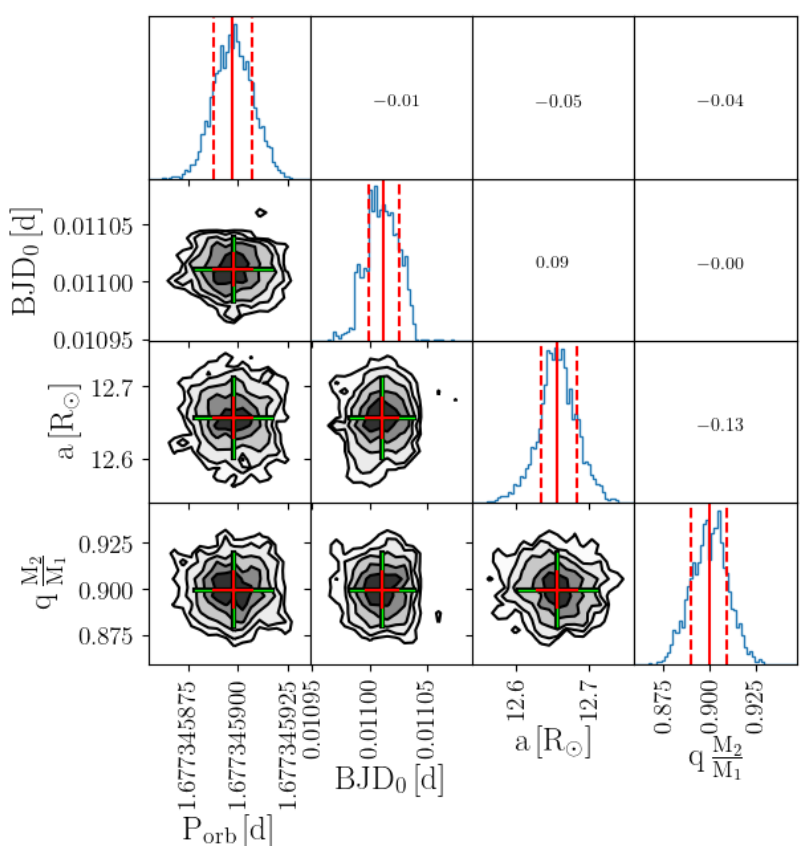

Fig. B.3. Marginalised posterior distributions for the orbital parameters. The median is shown by a solid vertical red line, the upper and lower bounds for $68.27 \%$ CI by dashed vertical red lines.

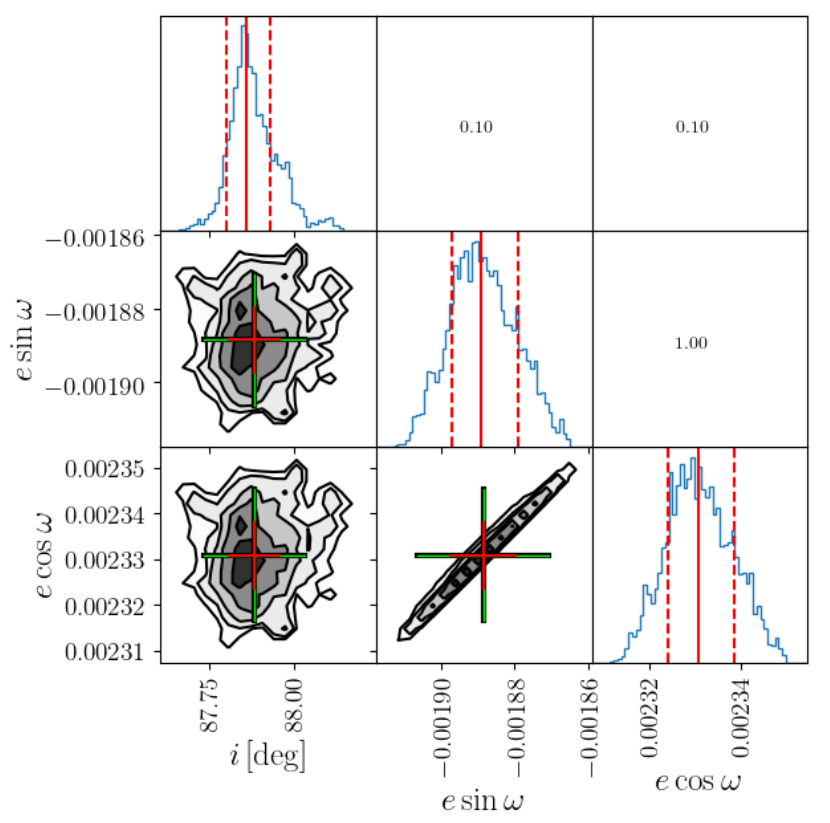

Fig. B.4. Marginalised posterior distributions for system parameters. The median is shown by a solid vertical red line, the upper and lower bounds for $68.27 \%$ CI by dashed vertical red lines. 